\title{
Energy-based variational modeling of adiabatic shear bands structure evolution
}

\author{
Shaopu Su, Laurent Stainier \\ Institut de Recherche en Génie Civil et Mécanique (GeM, UMR 6183 CNRS), École Centrale Nantes, 1 rue de la Noë, BP 92101 , F-44321 Nantes, France
}

A novel energy-based variational approach is proposed for modeling adiabatic shear band (ASB) structure evolution, including elasticity, work hardening, and heat conduction. Conservation laws are formulated as a mathematical optimization problem with respect to a limited set of scalars. Consequently, by means of canonical expressions of displacement and temperature, the bandwidth and the central temperature can be accurately computed as internal variables. Based on this thermo-mechanical coupled variational framework, we can verify the generality of the proposed analytical approach with respect to constitutive models, as illustrated through various thermal softening laws such as power laws or the popular Johnson-Cook model. In addition, accounting for work hardening and elasticity, we propose an effective (or macroscopic) thermo-elasto-viscoplastic model of the shear localization zone in transient regime. A new loading/unloading condition, stemming as a Kuhn-Tucker relation, is introduced for this variational model. The stress evolution and the capacity of the approach to handle cyclic loading are analyzed, presenting a very good correspondence with ASB simulations by finite element method.

\section{Keywords:}

Adiabatic shear band

Variational updates

Thermo-elasto-visco-plasticity

\section{Introduction}

Shear localization is a common phenomenon in ductile materials undergoing high strain rate loading. An adiabatic shear band (ASB) is a relatively narrow band, typically a few tens of micrometers in width, presenting large deformation and high temperature, occurring in various ductile materials (metals, polymers,...) where thermal softening is significant (Zener and Hollomon, 1944). The adjective adiabatic can actually be considered as a misnomer, since heat conduction plays a key role in their formation (Merzer, 1982; Wright, 2002). Although an ASB can appear as a strong displacement or velocity discontinuity at the macroscopic scale, it is actually a weak discontinuity (Oliver and Huespe, 2004), whose width and intensity are controlled by the balance between heat production from mechanical dissipation and heat diffusion by thermal conduction. One of the objectives of this work is to bridge the two descriptions, which are each relevant to different scales. Note also that other softening mechanisms than temperature can be at play: in particular, recent work (Rittel et al., 2008; Dolinski et al., 2010) has shown the important role of micro-mechanisms such as dynamic recrystallization, which could also be at the origin of ASB formation. In the present paper, we will nonetheless restrict ourselves to thermal softening.

Since first observed by Tresca in the 19th century, the problem of initiation, formation and propagation of adiabatic shear bands has drawn a great attention in the research community. ASBs play an important role in many manufacturing processes (forming, cutting) and in the resistance of 
structures under impact loading. The numerical treatment of these problems has been persistently hampered by difficulties in correctly representing the very strong localization of deformation (and temperature) associated to ASBs. When limiting the analysis to its mechanical aspects (e.g. using a locally adiabatic constitutive model), it is nowadays wellestablished that spurious mesh-dependence may appear, leading to a wide variety of regularization approaches on which we will not dwell here. But even when accounting for the complete coupled thermo-mechanical setting, in which framework the problem is well posed, the typically very small width of ASBs (of the order of the $\mu \mathrm{m}$ ) can cause serious difficulties. On the one hand, the mesh size required to resolve such characteristic length is often limiting in terms of modeling actual industrial problems, and on the other hand the very large deformations occurring in the localized shear zone typically lead to strong mesh distortion and associated numerical problems. Various approaches have been suggested in order to circumvent these meshrelated difficulties, among which: the use of mesh-free methods, or the introduction of discontinuities to represent shear bands. Mesh-free methods (see for example Li et al., 2002; Medyanik et al., 2007) may alleviate mesh distortion problem, but still require some level of adaptivity in order to capture characteristic shear band widths. Comparatively, the incorporation of discontinuities in the numerical model avoids the need to resolve such small length. Among this type of approach, we can note embedded discontinuities (Ortiz et al., 1987; Oliver et al., 1999), interface elements (Ortiz and Pandolfi, 1999; Yang et al., 2005), or the use of XFEM enrichment (Areias and Belytschko, 2007). Alternatively, discontinuities can be embedded directly into the constitutive model (Longère et al., 2003; Longère et al., 2005; Longère et al., 2009). Each of these discontinuous approaches has its advantages and inconveniences in terms of how they deal with mesh related problems and are able to accurately represent shear bands, but they share the common characteristic of relying on the choice of a shear band width parameter. Results of numerical simulations can vary significantly with this choice.

In this paper, we propose a novel energy-based variational approach for modeling ASB structure, including elasticity, work hardening, and heat conduction, yet avoiding the use of a mesh. Conservation laws are formulated as a mathematical optimization problem with respect to a limited set of scalars. Consequently, by means of canonical expressions of displacement and temperature (taken from Leroy and Molinari, 1992), the bandwidth and the central temperature can be accurately computed as internal parameters/variables. This variational approach has been successfully applied to steady-state ASBs (Su et al., 2014). In this paper, we consider the transient problem of evolution of ASB structure, from onset to steady-state, at least when the latter does exist. In our model, the evolving shear band width is thus dynamically computed, avoiding the delicate choice imposed on alternative methods listed above. Based on this thermo-mechanical coupled variational framework, we can verify the generality of the proposed analytical approach with respect to constitutive models, as illustrated through various thermal softening laws such as power laws or the popular Johnson-Cook model. In addition, accounting for work hardening and elasticity, we propose an effective (or macroscopic) thermo-elasto-viscoplastic model of the shear localization zone. A new loading/unloading condition, stemming as a Kuhn-Tucker relation, is introduced for this variational model. The stress evolution and the capacity of the approach to handle cyclic loading are analyzed, presenting a very good correspondence with ASB simulations by finite element method (FEM). Yet, the proposed variational approach, which can be seen as a Ritz-Galerkin method, involves only four scalar unknowns to describe the full shear band structure (strain and temperature), while FEM models typically involve at least hundreds of degrees of freedom, leading to a lesser computational cost for the Ritz-Galerkin approach. Finally, it provides in the end a macroscopic strong discontinuity model, with physicallymotivated rate-dependent laws relating shear stress and heat production to tangential displacement discontinuity, opening the way to further improvement of discontinuity-based approaches discussed above.

The paper is organized as follows. In Section 2, we briefly review the variational framework for thermomechanical boundary-value problems which was recently proposed by Yang et al. (2006). In Section 3, we describe the proposed approach in the context of non-hardening thermo-elasto-visco-plasticity. We first lay down the assumptions underlying the unidimensional shear band model used here, and the associated variational formulation. We then introduce the Ritz-Galerkin approach, based on canonical functions established by Leroy and Molinari (1992), and detail some algorithmic issues critical to the numerical model, introducing a predictor-corrector scheme, leading to an original average shear band yield criterion. This model is then validated by comparison with FEM solutions, and we then show that it can naturally be extended to arbitrary thermo-visco-plasticity models. In Section 4, we reformulate the variational model in order to account for hardening, taking as a practical example the classical Johnson and Cook (1983) model. This modified variational model is again validated against FEM results, and we then show that it can be used for arbitrary non-monotonous loading paths. We also discuss a couple of algorithmic aspects, such as the influence of an algorithmic parameter and the evaluation of the error associated to the average yield criterion. We finally draw some conclusions and perspectives.

\section{Variational constitutive updates}

In this section, we briefly review the variational framework for thermo-mechanical boundary-value problems which was recently proposed by Yang et al. (2006). For a broader overview of this incremental variational framework, the reader is also referred to Stainier (2013).

\subsection{Thermodynamic framework}

For the sake of clarity, the variational framework is presented here under the assumption of small strains and small displacements (linearized kinematics), although it 
can easily be extended to finite strains (Stainier, 2013). The thermodynamic state of a material point is then described by the engineering strain tensor $\boldsymbol{\varepsilon}$, the absolute temperature $T$, and suitable internal variables. Considering elasto(visco-) plastic behavior, the total strain is decomposed into elastic and plastic parts:

$\boldsymbol{\varepsilon}=\boldsymbol{\varepsilon}^{e}+\boldsymbol{\varepsilon}^{p}$.

The rate of plastic strain can be rewritten in terms of amplitude and direction as follows:

$\dot{\boldsymbol{\varepsilon}}^{p}=\dot{\bar{\varepsilon}}^{p} \boldsymbol{M}$

where $\boldsymbol{M}$ is a traceless normalized symmetric tensor:

$\operatorname{tr} \boldsymbol{M}=0 ; \quad \boldsymbol{M} \cdot \boldsymbol{M}=\frac{3}{2} ; \quad \boldsymbol{M}^{T}=\boldsymbol{M}$.

The cumulated, or equivalent, plastic strain is then defined by

$\dot{\bar{\varepsilon}}^{p}=\sqrt{\frac{2}{3} \dot{\boldsymbol{\varepsilon}}^{p} \cdot \dot{\boldsymbol{\varepsilon}}^{p}}$

In the case of classical von Mises $\left(J_{2}\right)$ plasticity, internal variables are then defined as the set $\left\{\boldsymbol{\varepsilon}^{p}, \bar{\varepsilon}^{p}\right\}$.

Let us now assume the existence of a Helmholtz free energy density, a state function of the material state. For metals, it is commonly admitted that such an energy is the sum of elastic, plastic and thermal energies:

$W\left(\boldsymbol{\varepsilon}, T, \boldsymbol{\varepsilon}^{p}, \bar{\varepsilon}^{p}\right)=W^{e}\left(\boldsymbol{\varepsilon}-\boldsymbol{\varepsilon}^{p}, T\right)+W^{p}\left(\boldsymbol{\varepsilon}^{p}, \bar{\varepsilon}^{p}, T\right)+W^{t}(T)$.

In the following, we will consider isotropic materials, with

$W^{e}\left(\boldsymbol{\varepsilon}^{e}, T\right)=\frac{1}{2} \kappa(T)\left(\operatorname{tr}\left[\boldsymbol{\varepsilon}^{e}\right]\right)^{2}+\mu(T)\left\|\operatorname{dev}\left[\boldsymbol{\varepsilon}^{e}\right]\right\|^{2}$,

where $\kappa$ and $\mu$ are the bulk and shear modulus, respectively. Note that we will not actually consider a temperature-dependence of elastic moduli in the following. The (Cauchy) stress tensor is obtained as the thermodynamic force conjugate to strain:

$\boldsymbol{\sigma}=\frac{\partial W}{\partial \boldsymbol{\varepsilon}}=\frac{\partial W^{e}}{\partial \boldsymbol{\varepsilon}^{e}}=\kappa \operatorname{tr}\left[\boldsymbol{\varepsilon}^{e}\right] \boldsymbol{\delta}+2 \mu \operatorname{dev}\left[\boldsymbol{\varepsilon}^{e}\right]$,

where $\boldsymbol{\delta}$ denotes the second-order identity tensor. The form of the stored plastic energy will be discussed below. Note at this point that an explicit dependence on the plastic strain tensor $\boldsymbol{\varepsilon}^{p}$ corresponds to kinematic hardening, with a backstress tensor given by

$\boldsymbol{\chi}=\frac{\partial W^{p}}{\partial \boldsymbol{\varepsilon}^{p}}\left(\boldsymbol{\varepsilon}^{p}, \bar{\varepsilon}^{p}, T\right)$

while dependence on the scalar cumulated plastic strain can be associated to isotropic hardening, with the yield stress

$g=\frac{\partial W^{p}}{\partial \bar{\varepsilon}^{p}}\left(\boldsymbol{\varepsilon}^{p}, \bar{\varepsilon}^{p}, T\right)$

A possible expression (see Miehe, 1995) for the stored thermal energy (heat storage capacity) is given by

$W^{t}(T)=\rho_{0} C_{0}\left[T-T_{0}-T \log \left(\frac{T}{T_{0}}\right)\right]$,

where $C_{0}$ is the specific heat capacity, $\rho_{0}$ the mass density, and $T_{0}$ a reference temperature. The entropy density is obtained from the free energy as the thermodynamic force conjugate to temperature:

$$
\begin{aligned}
\rho_{0} \eta= & -\frac{\partial W}{\partial T}\left(\boldsymbol{\varepsilon}, T, \boldsymbol{\varepsilon}^{p}, \bar{\varepsilon}^{p}\right)=-\frac{\partial W^{e}}{\partial T}\left(\boldsymbol{\varepsilon}^{e}, T\right)-\frac{\partial W^{p}}{\partial T}\left(\boldsymbol{\varepsilon}^{p}, \bar{\varepsilon}^{p}, T\right) \\
& +\rho_{0} C_{0} \log \left(\frac{T}{T_{0}}\right)
\end{aligned}
$$

where $\eta$ is the specific entropy. Accounting for the flow rule (2), the rate of the free energy can then be written as

$\dot{W}=\boldsymbol{\sigma} \cdot \dot{\boldsymbol{\varepsilon}}-[(\boldsymbol{\sigma}-\boldsymbol{\chi}) \cdot \boldsymbol{M}-g] \dot{\bar{\varepsilon}}^{p}-\rho_{0} \eta \dot{T}$

and we thus see that we can consider that the effective quantity

$y=(\boldsymbol{\sigma}-\boldsymbol{\chi}) \cdot \boldsymbol{M}-\mathrm{g}$

is thermodynamically conjugate to $\bar{\varepsilon}^{p}$.

In order to complete the framework, we introduce a dissipation (pseudo-)potential $\psi(y)$, from which evolution laws for internal variables are derived (Generalized Standard Material framework of Halphen and Nguyen, 1975):

$\dot{\bar{\varepsilon}}^{p}=\frac{\partial \psi}{\partial y}\left(y ; \bar{\varepsilon}^{p}, T\right)$

where arguments of $\psi$ after the semicolon describe a potential parametric dependence on the material state. The inverse relation can be obtained through a LegendreFenchel transform:

$\psi^{*}\left(\dot{\bar{\varepsilon}}^{p} ; \bar{\varepsilon}^{p}, T\right)=\sup _{y}\left[y \dot{\bar{\varepsilon}}^{p}-\psi\left(y ; \bar{\varepsilon}^{p}, T\right)\right]$

and

$y=\frac{\partial \psi^{*}}{\partial \dot{\bar{\varepsilon}}^{p}}\left(\dot{\bar{\varepsilon}}^{p} ; \bar{\varepsilon}^{p}, T\right)$

Convexity of $\psi$ (and subsequently of $\psi^{*}$ ) ensure nonnegativeness of dissipation $\mathcal{D}=y \dot{\bar{\varepsilon}}^{p}$. Specific expressions for $\psi^{*}$ will be discussed in the following sections. Evolution Eq. (16) can be rewritten as

$\frac{\partial}{\partial \dot{\bar{\varepsilon}} p}\left[\dot{W}+\psi^{*}\right]=0$

or, given the convexity of $\psi^{*}$

$\dot{\bar{\varepsilon}}^{p}=\arg \inf _{\dot{\bar{\varepsilon}}^{p}}\left[\dot{W}+\psi^{*}\right]$.

Similarly, we can write

$\boldsymbol{M}=\arg \inf _{\boldsymbol{M}}\left[\dot{W}+\psi^{*}\right]$,

which corresponds to the principle of maximum dissipation

$\boldsymbol{M}=\arg \sup _{\boldsymbol{M}} y \dot{\bar{\varepsilon}}^{p}$

Constraints (3) must of course be taken into account in optimization problem (19), yielding (Ortiz and Stainier, 1999)

$\boldsymbol{M}=\sqrt{\frac{3}{2}} \frac{\operatorname{dev}[\boldsymbol{\sigma}-\boldsymbol{\chi}]}{\|\operatorname{dev}[\boldsymbol{\sigma}-\boldsymbol{\chi}]\|}$ 


\subsection{Incremental variational principle}

The variational principle defined by (18) and (19) can be expressed in a time-incremental framework as follows (Yang et al., 2006):

$$
\begin{aligned}
\mathcal{W}_{n}\left(\boldsymbol{\varepsilon}_{n+1}, T_{n+1} ; \boldsymbol{\varepsilon}_{n}, T_{n}, \boldsymbol{\varepsilon}_{n}^{p}, \bar{\varepsilon}_{n}^{p}\right)= & \inf _{\bar{\varepsilon}_{n+1}^{p}, \boldsymbol{M}}\left[W\left(\boldsymbol{\varepsilon}_{n+1}, T_{n+1}, \boldsymbol{\varepsilon}_{n+1}^{p}, \bar{\varepsilon}_{n+1}^{p}\right)\right. \\
& -W\left(\boldsymbol{\varepsilon}_{n}, T_{n}, \boldsymbol{\varepsilon}_{n}^{p}, \bar{\varepsilon}_{n}^{p}\right)-\rho_{0} \eta_{n}\left(T_{n+1}-T_{n}\right) \\
& \left.+\int_{t_{n}}^{t_{n+1}} \psi^{*}\left(\frac{T_{n+1}}{T_{n}} \frac{\Delta \bar{\varepsilon}^{p}}{\Delta t} ; \bar{\varepsilon}^{p}\left(t^{\prime}\right), T\left(t^{\prime}\right)\right) d t^{\prime}\right],
\end{aligned}
$$

where subscripts $n$ and $n+1$ refer to instants $t_{n}$ and $t_{n+1}$, respectively, and $\Delta t=t_{n+1}-t_{n}, \Delta \bar{\varepsilon}^{p}=\bar{\varepsilon}_{n+1}^{p}-\bar{\varepsilon}_{n}^{p}$. In the above expression, the updated plastic strain tensor is given by the incremental flow rule:

$\boldsymbol{\varepsilon}_{n+1}^{p}=\boldsymbol{\varepsilon}_{n}^{p}+\Delta \bar{\varepsilon}^{p} \boldsymbol{M}$

In addition a consistent approximation to the integral of the dual dissipation potential $\psi^{*}$ must be provided, for example following the specific expression proposed in Stainier (2011):

$$
\begin{aligned}
\int_{t_{n}}^{t_{n+1}} & \psi^{*}\left(\frac{T_{n+1}}{T_{n}} \frac{\Delta \bar{\varepsilon}^{p}}{\Delta t} ; \bar{\varepsilon}^{p}\left(t^{\prime}\right), T\left(t^{\prime}\right)\right) d t^{\prime} \\
& \approx \Delta t\left[\frac{T_{n}}{T_{n+1}} \psi^{*}\left(\frac{T_{n+1}}{T_{n}} \frac{\Delta \bar{\varepsilon}^{p}}{\Delta t} ; \bar{\varepsilon}_{n+\alpha}^{p}, T_{n}\right)\right. \\
& \left.+\frac{\Delta T}{T_{n+1}} \psi^{*}\left(\frac{T_{n+1}}{T_{n}} \frac{\Delta \bar{\varepsilon}^{p}}{\Delta t} ; \bar{\varepsilon}_{n+\alpha}^{p}, T_{n+\alpha}\right)\right],
\end{aligned}
$$

where $\left\{\bar{\varepsilon}^{p}, T\right\}_{n+\alpha}=(1-\alpha)\left\{\bar{\varepsilon}^{p}, T\right\}_{n}+\alpha\left\{\bar{\varepsilon}^{p}, T\right\}_{n+1}$, with the algorithmic parameter $\alpha \in[0,1]$. The incremental energy defined in (22) can then be seen as an effective thermoelastic potential, in the sense that

$$
\begin{aligned}
\frac{\partial \mathcal{W}_{n}}{\partial \boldsymbol{\varepsilon}_{n+1}} & =\boldsymbol{\sigma}_{n+1}, \\
\frac{\partial \mathcal{W}_{n}}{\partial T_{n+1}} & =-\rho_{0} \Delta \eta+\Delta t \frac{\overline{\mathcal{D}}}{T_{n+1}},
\end{aligned}
$$

where $\Delta t \overline{\mathcal{D}} \approx y_{n+1} \Delta \bar{\varepsilon}^{p}$ is a consistent approximation of the energy dissipated by visco-plasticity over the time increment.

We can then define the following functional of displacement (denoted by $\boldsymbol{u}$ ) and temperature fields (Yang et al., 2006):

$\Phi_{n}\left(\boldsymbol{u}_{n+1}, T_{n+1}\right)=\int_{\Omega}\left[\mathcal{W}_{n}\left(\nabla \boldsymbol{u}_{n+1}, T_{n+1}\right)-\Delta t \mathcal{X}\left(-\frac{\nabla T_{n+1}}{T_{n+1}}\right)\right] d V$,

where we have dropped the dependence on the external and internal variable fields at $t_{n}$, for conciseness. In this expression, $\mathcal{X}$ is a Fourier potential such that the heat flux is given by

$\boldsymbol{q}=\frac{\partial \mathcal{X}}{\partial \boldsymbol{G}}(\boldsymbol{G})$ where $\boldsymbol{G}=-\frac{\nabla T}{T}$

(in the spirit of Biot, 1958). For example, in the case of an isotropic material with a heat conduction coefficient $\lambda$, we can write
$\mathcal{X}\left(\boldsymbol{G}_{n+1} ; T_{n}\right)=\frac{1}{2} \lambda T_{n} \boldsymbol{G}_{n+1} \cdot \boldsymbol{G}_{n+1}$.

Under the assumption of pure Dirichlet boundary conditions (extension to Neumann, or even mixed, boundary conditions is quite trivial, see Stainier, 2013), the incremental coupled thermo-mechanical boundary-value problem can then be stated as a variational principle:

$\left\{\boldsymbol{u}_{n+1}, T_{n+1}\right\}=\arg \inf _{\boldsymbol{u}_{n+1} \sup _{n+1}} \Phi_{n}\left(\boldsymbol{u}_{n+1}, T_{n+1}\right)$.

Indeed, Euler-Lagrange equations for this variational problem correspond to consistent incremental approximations of (quasistatic) mechanical balance equation and heat equation.

This variational formulation of coupled thermomechanical problems presents several advantages. First, it inherits the symmetry associated to all variational formulations, a property which is generally missing in conventional formulations of thermo-mechanical problems. The variational nature of the problem also allows to use tools from optimization theory and mathematical programming. In particular, we will use in the following sections a Ritz-Galerkin approach to derive an approximate solution to the thermo-mechanical problem of an adiabatic shear band.

\section{Variational modeling for shear bands without hardening}

\subsection{Variational shear band model}

In the sequel, following Leroy and Molinari (1992), we simplify the problem of a localized shear band by considering a layer of infinite length ( $x$-direction) and thickness $2 \mathrm{H}$ (y-direction), subject to a shearing velocity $\pm V_{0}$ at $y= \pm H$, as described in Fig. 1. We consider a plane-strain assumption, and isothermal boundary conditions: $T=T_{0}$ at $y= \pm H$. The displacement and temperature fields then reduce to $\boldsymbol{u}=u(y, t) \boldsymbol{e}_{x}$ and $T(y, t)$. The strain tensor reduces to $\boldsymbol{\varepsilon}(y, t)=\frac{1}{2} u_{y}(y, t)\left(\boldsymbol{e}_{x} \otimes \boldsymbol{e}_{y}+\boldsymbol{e}_{y} \otimes \boldsymbol{e}_{x}\right)$, and the temperature gradient reduces to $\nabla T=T_{y}(y, t) \boldsymbol{e}_{y}$. The incremental variational principle then takes the form

$$
\begin{aligned}
\left\{u_{n+1}(y), T_{n+1}(y)\right\}= & \arg \inf _{u_{n+1}} \sup _{T_{n+1}} \int_{-H}^{H}\left[\mathcal{W}_{n}\left(\boldsymbol{\varepsilon}_{n+1}, T_{n+1}\right)\right. \\
& \left.-\Delta t \mathcal{X}\left(-\frac{T_{y}\left(y, t_{n+1}\right)}{T_{n+1}(y)}\right)\right] d y,
\end{aligned}
$$

where $u_{n+1}(y)=u\left(y, t_{n+1}\right)$ and $T_{n+1}(y)=T\left(y, t_{n+1}\right)$.

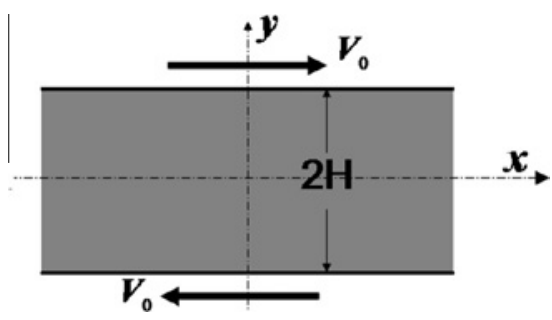

Fig. 1. Simplified 1D shear band problem (Leroy and Molinari, 1992). 
We will consider that the material in the shear band behaves according to an elasto-visco-plastic model, without hardening in a first approach. The strain rate is thus decomposed into an elastic and a (visco-)plastic part:

$\dot{\boldsymbol{\varepsilon}}=\left(\dot{\varepsilon}^{e}+\dot{\varepsilon}^{p}\right)\left(\boldsymbol{e}_{x} \otimes \boldsymbol{e}_{y}+\boldsymbol{e}_{y} \otimes \boldsymbol{e}_{x}\right)$.

The elastic strain rate is assumed to be uniform over the thickness, with

$\dot{\varepsilon}^{e}=\frac{1}{2} \frac{V_{0}{ }^{e}}{H}$,

where $V_{0}^{e}=V_{0}-V_{0}^{p}$ is the elastic part of the shearing velocity $V_{0}$. We can also define an elastic shearing displacement at the boundary

$U_{0}^{e}=\int_{0}^{t}\left(V_{0}-V_{0}^{p}\right) d \tau$

and the associated elastic strain

$\varepsilon^{e}=\frac{1}{2} \frac{U_{0}{ }^{e}}{H}$.

The elastic free energy is then given by

$W^{e}\left(U_{0}{ }^{e}\right)=\frac{\mu}{2}\left(\frac{U_{0}{ }^{e}}{H}\right)^{2}$.

In the absence of hardening, we will consider that the plastic free energy is identically null, while the thermal part is given by (10). Following Leroy and Molinari (1992), we consider a thermo-visco-plastic yield stress $\sigma_{y}$ exhibiting exponential thermal softening, combined with a powerlaw strain-rate sensitivity:

$\sigma_{y}=\sigma_{0} \exp \left[-\beta\left(\frac{T}{T_{0}}-1\right)\right]\left[1+\left(\frac{\dot{\bar{\gamma}}^{p}}{\dot{\gamma}_{0}}\right)^{m}\right]$,

where $\dot{\bar{\gamma}}^{p}$ is the (cumulated) plastic shear strain-rate, $\dot{\gamma}_{0}$ a reference strain-rate, $m$ a rate-dependency exponent, $\beta$ a thermal softening coefficient, and $\sigma_{0}$ the plastic yield stress (i.e. in the absence of viscous effects) at reference temperature. In the case of our simplified shear band model, we have

$\dot{\bar{\gamma}}^{p}(y, t)=\left|\dot{\gamma}^{p}(y, t)\right|=\sqrt{3} \dot{\bar{\varepsilon}}^{p}(y, t) \quad$ with

$\dot{\gamma}^{p}(y, t)=2 \dot{\varepsilon}^{p}(y, t)=\dot{u}_{y}(y, t)-\frac{V_{0}{ }^{e}(t)}{H}$.

Expression (37) can be recovered from the following dissipation pseudo-potential:

$$
\begin{aligned}
\psi^{*}\left(\dot{\bar{\varepsilon}}^{p} ; \bar{\varepsilon}^{p}, T\right)= & \sigma_{0} \exp \left[-\beta\left(\frac{T}{T_{0}}-1\right)\right] \dot{\bar{\varepsilon}}^{p} \\
& +\frac{\sigma_{0} \dot{\varepsilon}_{0}}{m+1} \exp \left[-\beta\left(\frac{T}{T_{0}}-1\right)\right]\left(\frac{\dot{\bar{\varepsilon}}^{p}}{\dot{\varepsilon}_{0}}\right)^{m+1},
\end{aligned}
$$

where $\dot{\varepsilon}_{0}=\dot{\gamma}_{0} / \sqrt{3}$ (we will also use $\tau_{0}=\sigma_{0} / \sqrt{3}$ ), and

$\sigma_{y}\left(\dot{\bar{\varepsilon}}^{p}, \bar{\varepsilon}^{p}, T\right)=\frac{\partial \psi^{*}}{\partial \dot{\bar{\varepsilon}}^{p}}\left(\dot{\bar{\varepsilon}}^{p} ; \bar{\varepsilon}^{p}, T\right)$.

Given the previous assumptions, the displacement profile across the shear band at time $t_{n+1}$ is given by

$u_{n+1}(y)=u_{n}(y)+v(y) \Delta t$, where $v( \pm H)= \pm V_{0}$ is known (imposed value, see above). The velocity profile can be split into an elastic and a plastic part:

$v(y)=V_{0}^{e} \frac{y}{H}+v^{p}(y)$

with $v^{p}( \pm H)= \pm V_{0}^{p}$ and $V_{0}^{e}=V_{0}-V_{0}^{p}$. Inspired by the work of Leroy and Molinari (1992), see also Wright and Ravichandran (1997), we will use the following canonical expressions for the plastic velocity and temperature profiles:

$v^{p}(y)=V_{0}^{p} \frac{\tanh \left(y / h_{V_{n+1}}\right)}{\tanh \left(H / h_{V_{n+1}}\right)}$,

$T_{n+1}(y)=T_{\max _{n+1}}-\left(T_{\max _{n+1}}-T_{0}\right) \frac{\ln \left(\cosh \left(y / h_{T_{n+1}}\right)\right)}{\ln \left(\cosh \left(H / h_{T_{n+1}}\right)\right)}$,

where $V_{0}^{p}, h_{V_{n+1}}, T_{\max _{n+1}}$ and $h_{T_{n+1}}$ are parameters to be determined. Note that the plastic shear rate is then given by

$\dot{\gamma}^{p}(y)=\frac{V_{0}^{p}}{h_{V_{n+1}}} \frac{1-\tanh \left(y / h_{V_{n+1}}\right)}{\tanh \left(H / h_{V_{n+1}}\right)}$.

As illustrated in Fig. $2, h_{V}$ and $h_{T}$ can respectively be interpreted as a kinematic and a thermal band width, while $T_{\max }$ represents the temperature at the center of the band.

The variational principle now becomes

$\inf _{V_{0}^{p}, h_{V_{n+1}} \sup _{\max _{n+1}}, h_{T_{n+1}}} \Phi_{n}\left(u_{n+1}\left(V_{0}^{p}, h_{V_{n+1}}\right), T_{n+1}\left(T_{\max _{n+1}}, h_{T_{n+1}}\right)\right)$,

where we emphasized that the displacement and temperature profiles are described by four scalar parameters. In practice, it appears useful to perform this optimization in two steps. First, we can compute the derivative of the functional $\Phi_{n}$ with respect to $V_{0}^{p}$, and evaluate it at $V_{0}^{p}=0^{ \pm}$ (which is a point of non-regularity of the functional, linked to the absolute value in the expression of $\psi^{*}$ ). The derivation is detailed in Appendix A, and yields:

$\frac{\partial \Phi_{n}}{\partial V_{0}^{p}}\left(V_{0}^{p}=0^{ \pm}\right)=2 \Delta t\left[-\tau_{\mathrm{tr}} \pm \bar{\tau}_{0_{n}}\right]$

where the trial shear stress $\tau_{\mathrm{tr}}$ is given by

$\tau_{\mathrm{tr}}=\mu \frac{U_{n}^{e}+V_{0} \Delta t}{H}$

and the average yield stress $\bar{\tau}_{0_{n}}$ by

$\bar{\tau}_{0_{n}}=\frac{\tau_{0}}{2} \int_{-H}^{H} \frac{1-\tanh \left(y / h_{V_{n+1}}\right)}{h_{V_{n+1}} \tanh \left(H / h_{V_{n+1}}\right)} \exp \left[-\beta\left(\frac{T_{n}(y)}{T_{0}}-1\right)\right] d y$.

Considering the convexity of $\Phi_{n}$ with respect to $V_{0}^{p}$, if $\left|\tau_{\text {tr }}\right| \leqslant \bar{\tau}_{0_{n}}$, then the optimum with respect to $V_{0}^{p}$ will clearly be at $V_{0}^{p}=0$ and the increment is elastic. If $\tau_{\mathrm{tr}}>\bar{\tau}_{0_{n}}$, then the optimum will correspond to a positive value of $V_{0}^{p}$, while if $\tau_{\mathrm{tr}}<-\bar{\tau}_{0_{n}}$, it will correspond to a negative value of $V_{0}^{p}$. This is illustrated at Fig. 3, where $\bar{V}_{0}^{p}=\left|V_{0}^{p}\right|$ with $V_{0}^{p}=\operatorname{sign}\left(\tau_{\mathrm{tr}}\right) \bar{V}_{0}^{p}$.

In the case of an elastic increment $\left(\left|\tau_{\text {tr }}\right| \leqslant \bar{\tau}_{0_{n}}\right)$, optimization with respect to $h_{V_{n+1}}$ is meaningless, since $V_{0}^{p}$ appears as a factor in expression (43). Thermal quantities 

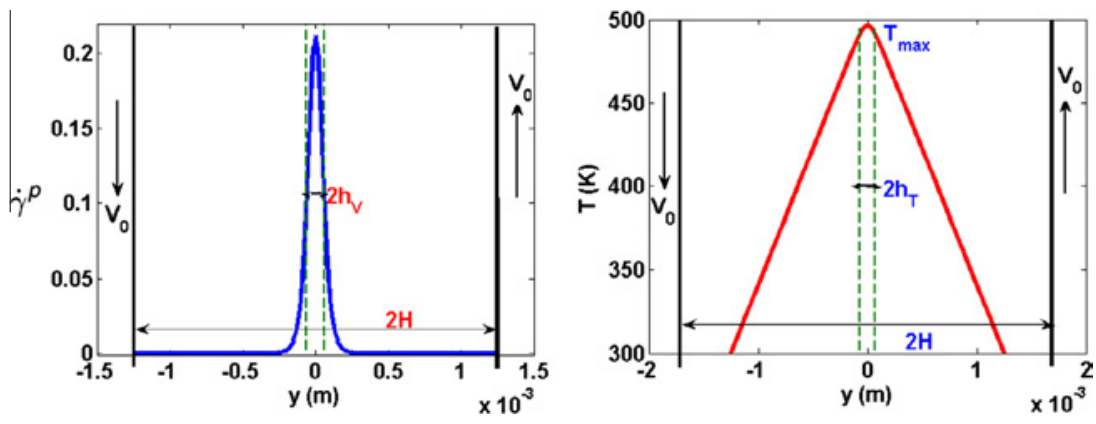

Fig. 2. Canonical profiles of shear strain rate and temperature $(H=1.25 \mathrm{~mm})$.

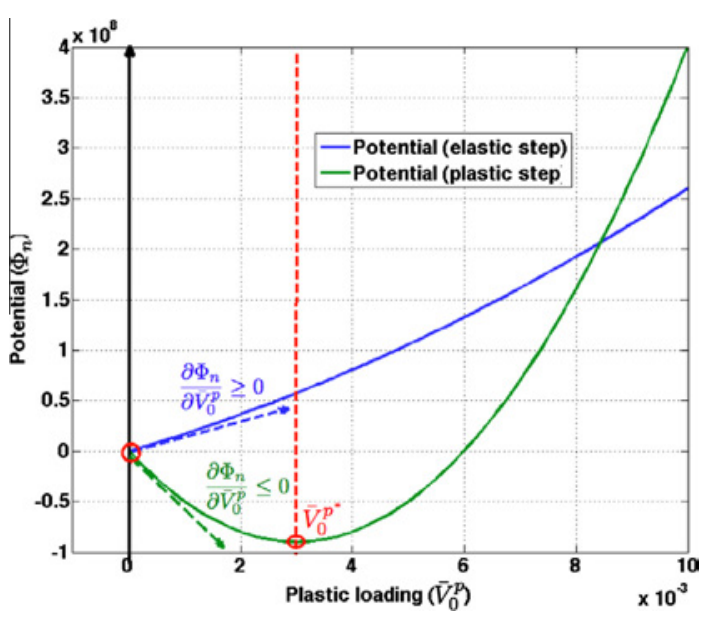

Fig. 3. Optimization of incremental functional with respect to plastic velocity amplitude.

must still be determined, since conduction will lead to some heat diffusion. The problem thus takes the form

if $\left|\tau_{\mathrm{tr}}\right| \leqslant \bar{\tau}_{0_{n}}: V_{0}^{p}=0, h_{V_{n+1}}=h_{V_{n}}$,

$\left\{T_{\max _{n+1}}, h_{T_{n+1}}\right\}=\arg \sup _{T_{\max }, h_{T}} \Phi_{n}\left(u_{n+1}\left(V_{0}^{p}, h_{V_{n+1}}\right), T_{n+1}\left(T_{\max }, h_{T}\right)\right)$.

Note that the solution to this problem will lead to a general decrease of temperature within the band, and thus, assuming that yield stress always decreases with temperature, the condition $\left|\tau_{n+1}\right| \leqslant \bar{\tau}_{0_{n+1}}$ will remain verified. In the case of a visco-plastic increment $\left(\left|\tau_{\mathrm{tr}}\right|>\bar{\tau}_{0_{n}}\right)$, the general problem (46) must be solved. In practice, this optimization problem is solved through an alternated directions approach. The algorithm followed at each time increment thus goes as follows:

1. Initialization $(k=0): V_{0,0}^{p}=0, h_{V_{n+1,0}}=h_{V_{n}}, T_{\max _{n+1,0}}=$ $T_{\max _{n}}, h_{T_{n+1,0}}=h_{T_{n}}$.

2. If $\left|\tau_{\mathrm{tr}}\right| \leqslant \bar{\tau}_{0_{n}}$, solve problem (50), and then proceed to next time increment (i.e. exit this algorithm). Otherwise, proceed to step 3.

3. At fixed $h_{V_{n+1, k}}, T_{\max _{n+1, k}}, h_{T_{n+1, k}}$, solve

$$
V_{0, k+1}^{p}=\arg \inf _{V_{0}^{p}} \Phi_{n}\left(u_{n+1}\left(V_{0}^{p}, h_{V_{n+1, k}}\right), T_{n+1}\left(T_{\max _{n+1, k}}, h_{T_{n+1, k}}\right)\right) .
$$

4. At fixed $V_{0, k+1}^{p}$, solve

$$
\begin{aligned}
& \left\{h_{V_{n+1, k+1}}, T_{\max _{n+1, k+1}}, h_{T_{n+1, k+1}}\right\} \\
& \quad=\arg \inf _{h_{V}} \sup _{T_{\max }, h_{T}} \Phi_{n}\left(u_{n+1}\left(V_{0, k+1}^{p}, h_{V}\right), T_{n+1}\left(T_{\max }, h_{T}\right)\right) .
\end{aligned}
$$

5. Check convergence (e.g. $\left|V_{0, k+1}^{p}-V_{0, k}^{p}\right|<\epsilon\left|V_{0,1}^{p}\right|$ ). If criterion is not met, loop to step $3(k \leftarrow k+1)$, otherwise proceed to next time increment (i.e. exit this algorithm).

Note that in early loading stages, localization may not be marked, and we thus enforce upper bounds to shear band width parameters: $h_{V} \leqslant 2.5 H$ (and if the bound is reached, $\left.h_{T}=h_{V}\right)$.

\subsection{Numerical results}

In order to numerically validate the proposed approach, we consider the same material as in Leroy and Molinari (1992), with a yield stress given by (37) and material parameters given in Table 1 . We start with a shear band formed at $V_{0}=0.011 \mathrm{~m} / \mathrm{s}$, with $H=1.25 \times 10^{-3} \mathrm{~m}$. Although Leroy and Molinari found an analytical solution for the steady-state problem, no such result exists for the transient regime. Therefore, a Finite Element model is used to establish a reference solution (see Appendix B for more details about this variational FE formulation). Fig. 4 presents the evolution of velocity and temperature profiles within the shear band, as obtained by the FE approach. Each curve represents a profile at a given time, and is also color-coded in function of magnitude. We can see that, as time increases, the velocity profile changes from a linear form (uniform shear strain rate) to a non-linear form (localized shear strain rate), finally reaching a steady state at time $t=0.2 \mathrm{~s}$. Simultaneously, temperature increases in the shear band's center, finally reaching a value $T_{\max }=496 \mathrm{~K}$ (plot shows temperature increase with

Table 1

Material properties for HY-100 steel.

\begin{tabular}{lllll}
\hline$E(\mathrm{GPa})$ & $v$ & $\rho_{0}\left(\mathrm{~kg} / \mathrm{m}^{3}\right)$ & $C_{0}(\mathrm{~J} / \mathrm{kg} / \mathrm{K})$ & $\lambda(\mathrm{W} / \mathrm{m} / \mathrm{K})$ \\
\hline 217.5 & 0.3 & 7800 & 500 & 54 \\
$\tau_{0}(\mathrm{MPa})$ & $\beta$ & $\dot{\gamma}_{0}\left(\mathrm{~s}^{-1}\right)$ & $m$ & $T_{0}(\mathrm{~K})$ \\
\hline 500 & 0.33 & 1000 & 0.012 & 300 \\
\hline
\end{tabular}



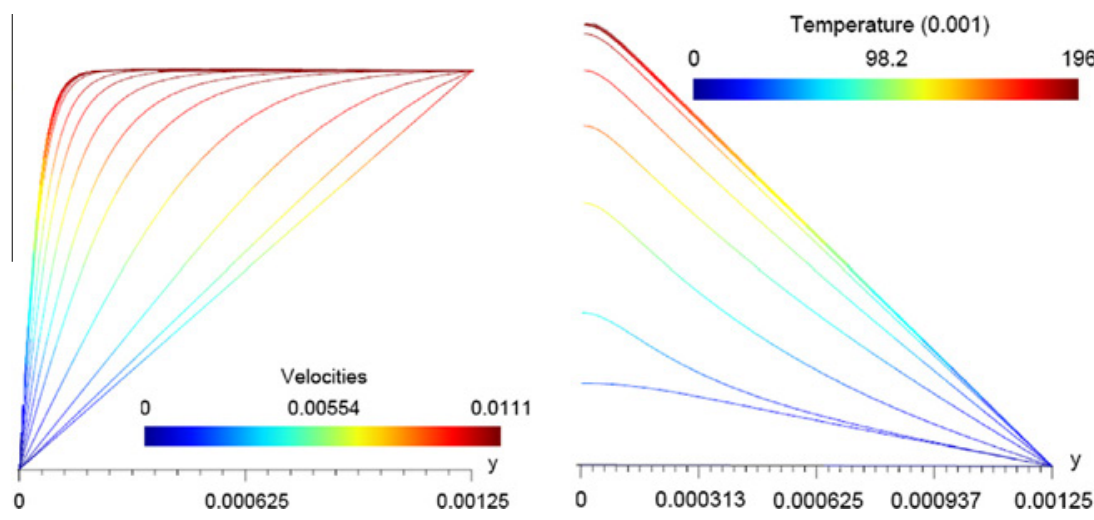

Fig. 4. Evolution of velocity and temperature profiles by FEM $\left(V_{0}=0.011 \mathrm{~m} / \mathrm{s}\right)$.
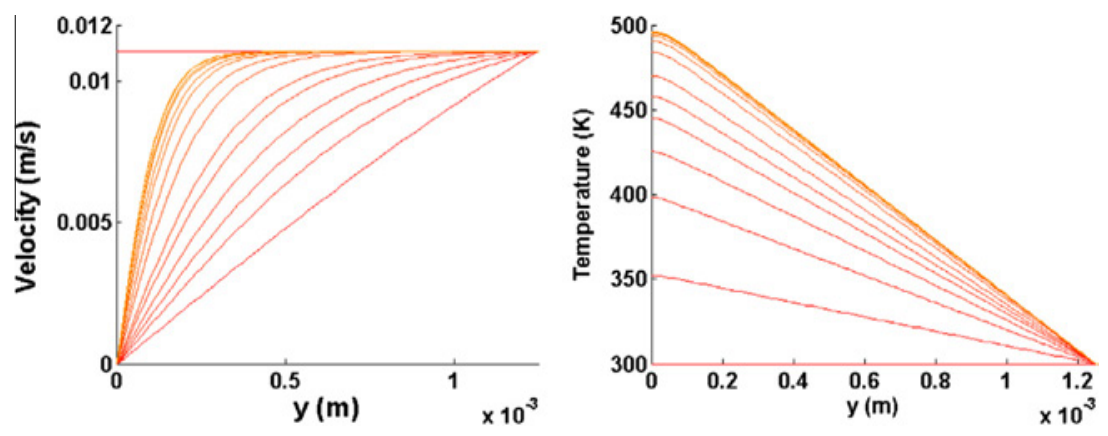

Fig. 5. Evolution of velocity and temperature profiles by Ritz-Galerkin method $\left(V_{0}=0.011 \mathrm{~m} / \mathrm{s}\right)$.

respect to $T_{0}$ ) at steady state. Outside of the central zone corresponding to the shear localization domain, the temperature varies linearly (constant gradient) when steadystate has been reached. We can already note that in the early stages of the transient regime, the temperature profile is more complex (coexistence of convex and concave parts). Note also that, despite the uni-dimensional nature of the problem, these FE simulations are costly in computation time, because they require a very fine mesh in the localization zone and small time increments for ensuring convergence.

Fig. 5 shows the evolution of profiles as obtained by the proposed variational Ritz-Galerkin approach (constant time increment $\Delta t=10^{-4} \mathrm{~s}$ ). These results are consistent with those obtained by FE. The main difference occurs for the temperature profile in early stages: the canonical function that we use is unable to represent mixed curvature profiles observed in the FE response. Despite this limitation, the variational method is able to provide a very good estimation of stress, as shown in Fig. 6. This figure plots the time evolution of shear stress within the band, for both FE and variational approaches. A semi-logarithmic scale was used to emphasize the existence of an elastic phase before the visco-plastic phase.

The above numerical simulations can be repeated with a faster loading rate imposed by a velocity jump $V_{0}=0.1 \mathrm{~m} / \mathrm{s}$. The resulting shear stress evolution is shown in Fig. 7, comparing both FE and Ritz-Galerkin approaches. The difference between the two approaches is more

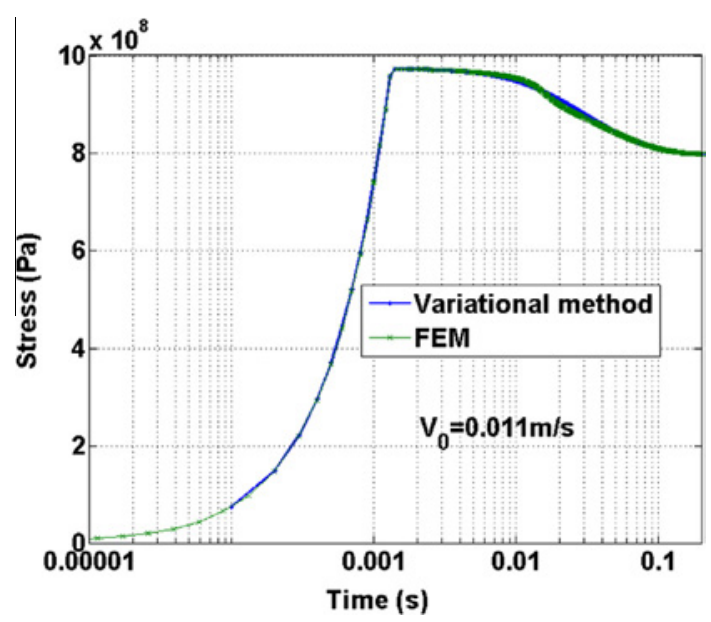

Fig. 6. Evolution of shear stress at $V_{0}=0.011 \mathrm{~m} / \mathrm{s}$.

marked (this can be traced to a stronger difference in the transient temperature profile at early stages), but both methods converge towards the same steady-state solution.

Aside of stress, we can also look at the evolution of the four scalar unknowns parameterizing the solution in the variational Ritz-Galerkin approach. For example, Fig. 8 shows the evolution of the ratio $\left|V_{0}^{p}\right| /\left|V_{0}\right|$, which measures the global amplitude of plastic strain rate in the band. We can see that the transition from elastic $\left(V_{0}^{p}=0\right)$ to 


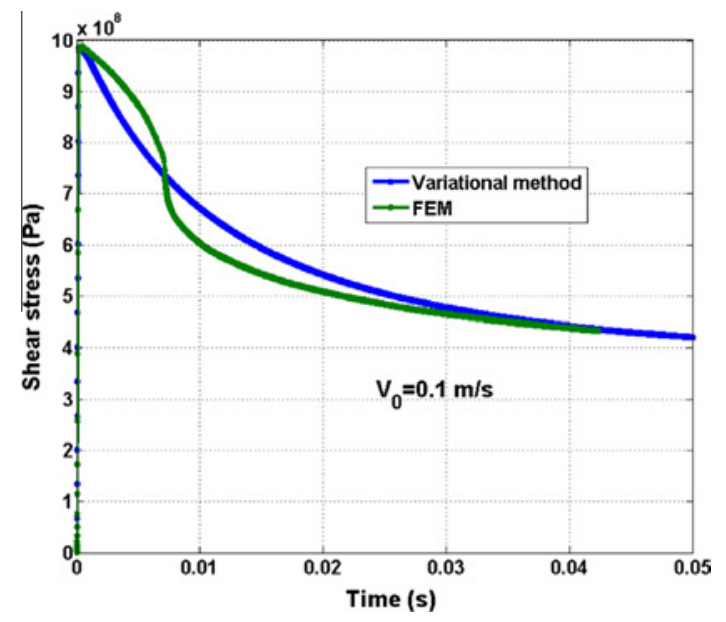

Fig. 7. Evolution of shear stress at $V_{0}=0.1 \mathrm{~m} / \mathrm{s}$.

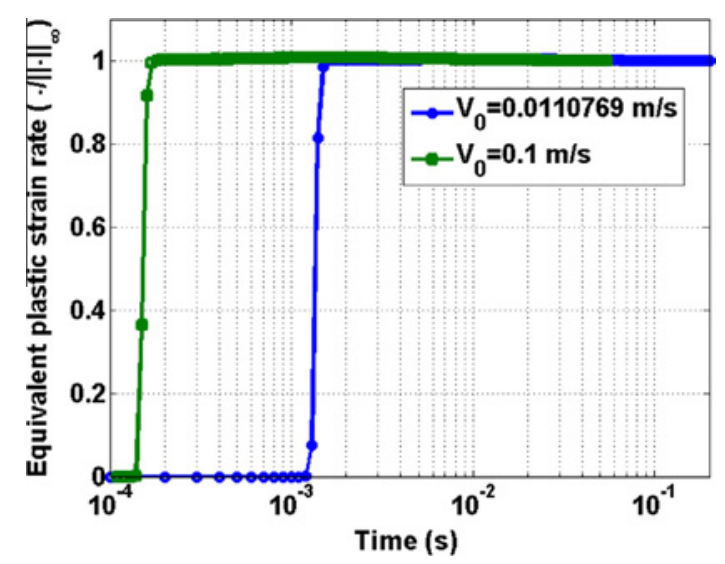

Fig. 8. Evolution of global plastic strain rate (relative) amplitude $\left|V_{0}^{p}\right| /\left|V_{0}\right|$.

predominantly (visco-)plastic $\left(V_{0}^{p} \simeq V_{0}\right)$ occurs in a few time increments. Of course, the transition also occurs at a later time for slow loading than for fast loading. In order to compare band width unknowns $h_{V}$ and $h_{T}$ with FE results, we must define a way to compute them from discrete profiles:

$h_{V_{\mathrm{FE}}}: V_{\mathrm{FE}}\left(h_{V_{\mathrm{FE}}}\right)=V_{0} \tanh (1)$,

$h_{T_{\mathrm{FE}}}: T_{\mathrm{FE}}\left(h_{T_{\mathrm{FE}}}\right)=T_{\max }-\left(T_{\max }-T_{0}\right) \frac{\log [\cosh (1)]}{\frac{H}{h_{T_{\mathrm{FE}}}}-\log (2)}$,

where we have assumed a priori that $h_{V} \ll H$ and $h_{T} \ll H$. Since actual profiles obtained by the FE approach may be quite different from canonical functions, these estimations should not be considered as true measures of shear band width, but seen as a way to qualitatively compare both approaches. Regarding the parameter $T_{\max }$, it will simply be taken as the temperature at the center of the band. Fig. 9 shows the evolution of the kinematic and thermal shear band widths with time, for both variational RitzGalerkin and FE approaches. Keeping in mind the above remarks on the computation of those width from FE results, the agreement is reasonably good. The localization

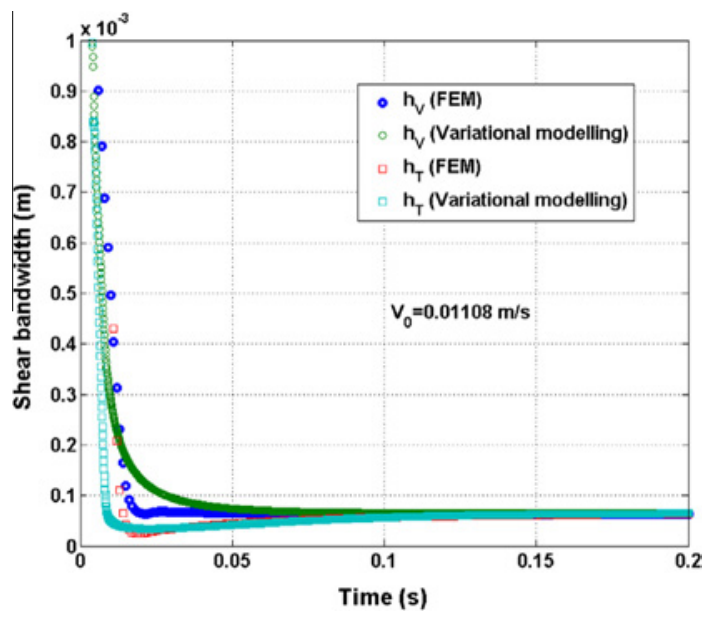

Fig. 9. Evolution of shear band widths $\left(V_{0}=0.011 \mathrm{~m} / \mathrm{s}\right)$

phase is well captured, and all curves converge towards a unique value (of $65 \mu \mathrm{m}$ ), in accordance with the analytical solution of Leroy and Molinari (1992). Fig. 10 shows the evolution of temperature at the center of the band, where it is maximal. Again, the agreement is quite good, the main differences occurring in the early stages as already pointed out. These results show that the variational Ritz-Galerkin approach proposed here does indeed offer a valid alternative to FE computations. For example, it can be used to predict the effect of shearing velocity on stress evolution, as illustrated in Fig. 11.

The main motivation for including elasticity in our variational model is to be able to account for elastic unloading stages. We thus proceed to test the variational method on a case of cyclic loading: $V_{0}=0.1 \mathrm{~m} / \mathrm{s}$ for $0 \leqslant t \leqslant 0.005 \mathrm{~s}$, followed by a reverse loading phase at $V_{0}=-0.1 \mathrm{~m} / \mathrm{s}$ for $0.005 \mathrm{~s} \leqslant t \leqslant 0.015 \mathrm{~s}$, and back to $V_{0}=0.1 \mathrm{~m} / \mathrm{s}$ for $t>0.015 \mathrm{~m} / \mathrm{s}$. The resulting stress evolution, both for $\mathrm{FE}$ and variational Ritz-Galerkin approaches, is shown in Fig. 12. Once more, small differences appear, especially in the early transient stage, but the overall agreement is good, keeping in mind that the Ritz-Galerkin approach involves 4 scalar unknowns (compared to hundreds of degrees of freedom for the FE approach).

\subsection{Extension to general constitutive models}

The Ritz-Galerkin approach described above can be applied to arbitrary thermo-visco-plasticity models. Although they do not correspond anymore to analytical solutions, canonical expressions (43) and (44) can still be used. For example, let us consider a power-law thermal softening model:

$\sigma_{y}=\sigma_{0}\left(\frac{T}{T_{0}}\right)^{-k}\left[1+\left(\frac{\dot{\bar{\varepsilon}}^{p}}{\dot{\varepsilon}_{0}}\right)^{m}\right]$

corresponding to the following dissipation pseudopotential:

$\psi^{*}\left(\dot{\bar{\varepsilon}}^{p} ; T\right)=\sigma_{0}\left(\frac{T}{T_{0}}\right)^{-k} \dot{\bar{\varepsilon}}^{p}+\frac{\sigma_{0} \dot{\varepsilon}_{0}}{m+1}\left(\frac{T}{T_{0}}\right)^{-k}\left(\frac{\dot{\bar{\varepsilon}}^{p}}{\dot{\bar{\varepsilon}}_{0}}\right)^{m+1}$. 


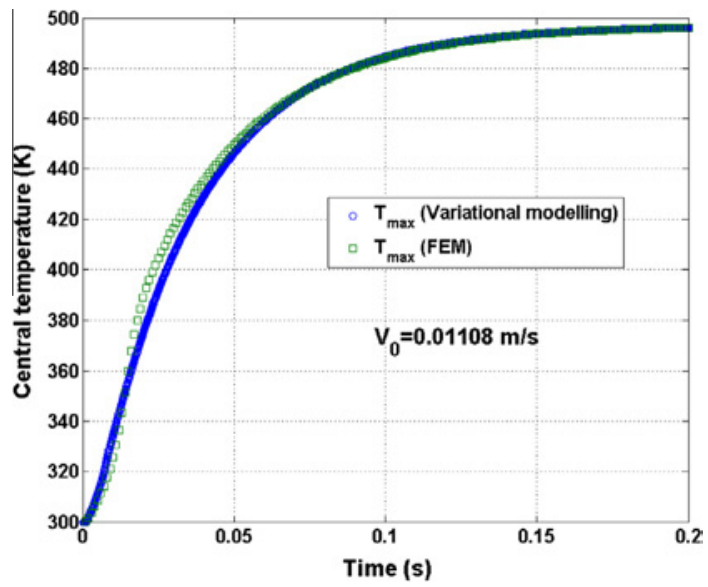

Fig. 10. Evolution of central temperature $\left(V_{0}=0.011 \mathrm{~m} / \mathrm{s}\right)$.

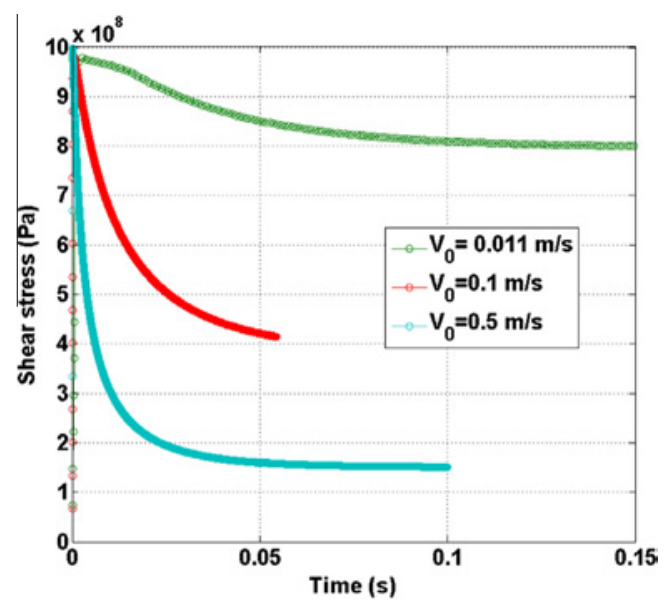

Fig. 11. Shear stress evolution for various shearing velocities.

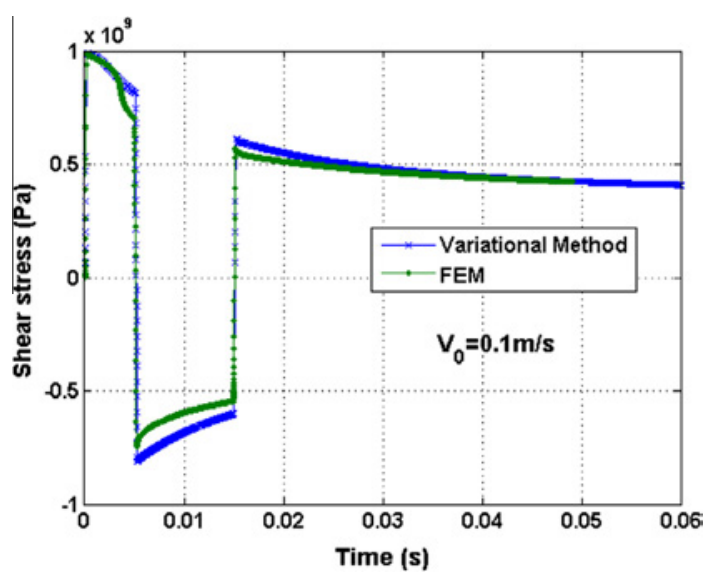

Fig. 12. Evolution of shear stress evolution under cyclic loading.

The Ritz-Galerkin variational approach can then be used, simply using the above expression in variational problem (46). For example, Fig. 13 shows the evolution of stress

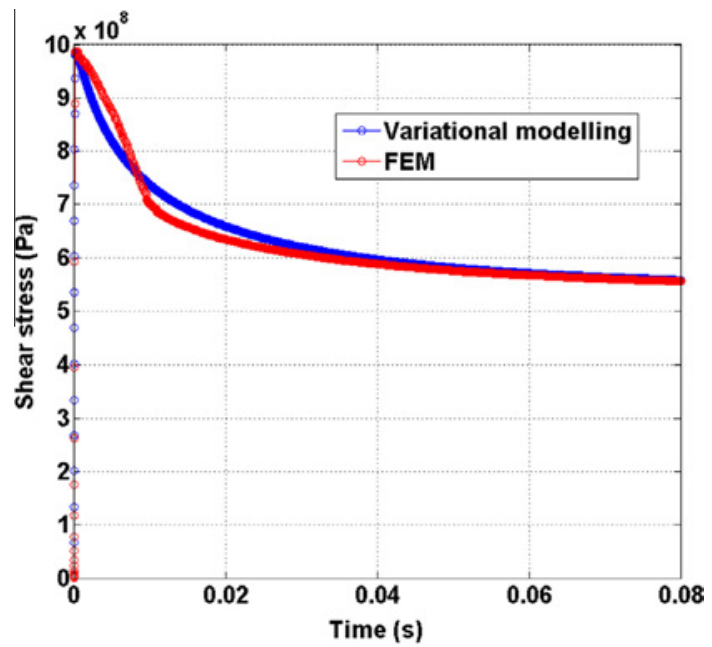

Fig. 13. Evolution of shear stress for a power-law thermal softening model $\left(V_{0}=0.1 \mathrm{~m} / \mathrm{s}\right)$.

Table 2

Material properties for CRS 1018 steel (Clifton and Molinari, 1983).

\begin{tabular}{lllll}
\hline$E(\mathrm{GPa})$ & $v$ & $\rho_{0}\left(\mathrm{~kg} / \mathrm{m}^{3}\right)$ & $C_{0}(\mathrm{~J} / \mathrm{kg} / \mathrm{K})$ & $\lambda(\mathrm{W} / \mathrm{m} / \mathrm{K})$ \\
\hline 217.5 & 0.3 & 7800 & 500 & 54 \\
$\sigma_{0}(\mathrm{MPa})$ & $k$ & $\dot{\varepsilon}_{0}\left(\mathrm{~s}^{-1}\right)$ & $m$ & $T_{0}(\mathrm{~K})$ \\
\hline 866 & 0.38 & 577 & 0.012 & 300 \\
\hline
\end{tabular}

for such a model, with parameters taken from Clifton and Molinari (1983) and listed in Table 2, in a band with a shearing velocity $V_{0}=0.1 \mathrm{~m} / \mathrm{s}\left(H=1.25 \times 10^{-3} \mathrm{~m}\right)$. The solution obtained by Ritz-Galerkin is compared to that obtained by FE, and we observe that the agreement between both remains good. The evolution of internal parameters $\left(V_{0}^{p}, h_{V}, h_{T}, T_{\max }\right)$, not shown here, follows the same trends as for the exponential softening model described above.

\section{Variational modeling for shear bands with hardening}

In most metallic materials, the formation of shear localization is the result of a competition between work hardening and thermal softening. Localized shear bands will appear when thermal softening takes over work hardening, and it is thus critical to take the latter into account for obtaining reliable predictions for ASB. In the following, we will illustrate how to include hardening in our variational approach, using the widely popular Johnson-Cook (JC) model.

\subsection{Johnson-Cook model}

The thermo-visco-plastic flow stress model proposed by Johnson and Cook (1983) is given by

$\sigma_{y}\left(\dot{\bar{\varepsilon}}^{p}, \bar{\varepsilon}^{p}, T\right)=\left(A+B\left(\bar{\varepsilon}^{p}\right)^{n}\right)\left[1+C \log \left(\frac{\dot{\bar{\varepsilon}}^{p}}{\dot{\bar{\varepsilon}}_{0}}\right)\right]\left(1-\theta^{* q}\right)$, 
where the non-dimensional temperature $\theta^{*}$ is defined as

$\theta^{*}= \begin{cases}0 & \text { if } T \leqslant T_{0} \\ \frac{T-T_{0}}{T_{m}-T_{0}} & \text { if } T_{0}<T \leqslant T_{m} \\ 1 & \text { if } T>T_{m} .\end{cases}$

Parameters $A, B, C, n, q$ are material constants, $\dot{\varepsilon}_{0}$ is a reference effective strain rate, $T_{m}$ is the melting temperature, and $T_{0}$ is the reference temperature.

This model can be described in terms of a plastic stored energy potential and a dissipation potential. The plastic stored energy potential is given by

$W^{p}\left(\bar{\varepsilon}^{p}, T\right)=\left(A_{s} \bar{\varepsilon}^{p}+\frac{B_{s}}{n+1}\left(\bar{\varepsilon}^{p}\right)^{n+1}\right)\left(1-\theta^{* q}\right)$.

The dissipation potential is given by

$$
\begin{aligned}
\psi^{*}\left(\dot{\bar{\varepsilon}}^{p} ; \bar{\varepsilon}^{p}, T\right)= & \left(A_{d}+B_{d}\left(\bar{\varepsilon}^{p}\right)^{n}\right)\left[\dot{\bar{\varepsilon}}^{p}+C \dot{\varepsilon}_{0}\left(\dot{\varepsilon}^{*} \log \left(\dot{\varepsilon}^{*}\right)-\dot{\varepsilon}^{*}+1\right)\right] \\
& \times\left(1-\theta^{* q}\right),
\end{aligned}
$$

where $\dot{\varepsilon}^{*}=\dot{\bar{\varepsilon}}^{p} / \dot{\varepsilon}_{0}$. Parameters $A_{s}, B_{s}, A_{d}$ and $B_{d}$ correspond to stored and dissipative parts of parameters $A$ and $B$ of the original Johnson-Cook model, with $A=A_{s}+A_{d}$ and $B=B_{s}+B_{d}$. Relative values of $A_{s}, B_{s}$ and $A_{d}, B_{d}$ control the fraction of plastic work power actually transformed into heat (so-called Taylor-Quinney coefficient).

\subsection{Variational shear band model}

In the variational model described in Section 3.1, we parameterized the plastic strain rate profile through the use of a canonical function. Since parameter $h_{V}$ varies with time in an arbitrary fashion, it is not possible to obtain an analytical expression of the (equivalent) plastic strain profile, although this would be necessary to account for (isotropic) hardening. Thus, in order to apply a Ritz-Galerkin approach to models such as JC, we need to modify our description of the shear band. We keep the uniform elastic strain adopted before, see Eq. (35), but now parameterize the equivalent plastic strain, instead of the plastic strain rate:

$\bar{\gamma}^{p}(y)=\frac{\bar{U}_{0}^{p}}{h_{V}} \frac{1-\tanh ^{2}\left(y / h_{V}\right)}{\tanh \left(H / h_{V}\right)}$,

where $\bar{U}_{0}^{p}$ and $h_{V}$ are parameters which vary with time. The equivalent plastic strain rate for a time increment $\left[t_{n}, t_{n+1}\right]$ is then given by

$\dot{\bar{\gamma}}^{p}(y)=\frac{\bar{\gamma}_{n+1}^{p}(y)-\bar{\gamma}_{n}^{p}(y)}{\Delta t}$

The elastic strain is computed from an elastic displacement jump:

$U_{0, n+1}^{e}=U_{0, n}^{e}+V_{0} \Delta t-\alpha\left(\bar{U}_{0, n+1}^{p}-\bar{U}_{0, n}^{p}\right)$,

where $\alpha= \pm 1$, and with $\bar{U}_{0, n+1}^{p} \geqslant \bar{U}_{0, n}^{p}$. In this incremental setting, we could alternatively define our parameter as $\Delta U_{0}^{p}=\alpha\left(\bar{U}_{0, n+1}^{p}-\bar{U}_{0, n}^{p}\right)$ (without sign restriction on $\left.\Delta U_{0}^{p}\right)$, with $\bar{U}_{0, n+1}^{p}=\bar{U}_{0, n}^{p}+\left|\Delta U_{0}^{p}\right|$.
Regarding the temperature profile, we still use expression (44). The variational principle then takes the following form

$\inf _{\Delta U_{0}^{p}, h_{V_{n+1}}} \sup _{\max _{n+1}, h_{T_{n+1}}} \Phi_{n}\left(\Delta U_{0}^{p}, h_{V_{n+1}}, T_{\max _{n+1}}, h_{T_{n+1}}\right)$.

As before, this variational problem is better solved by an alternate directions optimization method, with a starting point at $\Delta U_{0}^{p}=0$. Following the same line of reasoning as before, we obtain

$\frac{\partial \Phi_{n}}{\partial \Delta U_{0}^{p}}\left(\Delta U_{0}^{p}=0^{ \pm}\right)=2\left[-\tau_{\mathrm{tr}} \pm \bar{\tau}_{0_{n}}\right]$

and we recover the same predictor-corrector algorithm as in the non-hardening case. The whole algorithmic approach adopted in the non-hardening case can thus be used here as well, simply replacing $V_{0}^{p}$ by $\Delta U_{0}^{p}$. The main difference is that, because of variations of $h_{V}$ between $t_{n}$ and $t_{n+1}$, it may happen that expression (59) lead to negative values of $\dot{\bar{\gamma}}^{p}$ for some values of $y$. For these values of $y$, we use $\dot{\bar{\gamma}}^{p}=0$ in evaluating $\Phi_{n}$ and its derivatives. This is nonetheless a minor side effect of our choice of parameterization, necessary to account for hardening, and which does not have any visible effect on results as illustrated below.

\subsection{Numerical results}

In order to validate the above approach for ASB models with hardening, we will apply the Ritz-Galerkin formula-

Table 3

Material properties for Ti-6Al-4V.

\begin{tabular}{lllll}
\hline$E(\mathrm{GPa})$ & $v$ & $\rho_{0}\left(\mathrm{~kg} / \mathrm{m}^{3}\right)$ & $C_{0}(\mathrm{~J} / \mathrm{kg} / \mathrm{K})$ & $\lambda(\mathrm{W} / \mathrm{m} / \mathrm{K})$ \\
\hline 114 & 0.3 & 4428 & 580 & 6.7 \\
$A_{d}(\mathrm{MPa})$ & $A_{s}(\mathrm{MPa})$ & $B_{d}(\mathrm{MPa})$ & $B_{s}(\mathrm{MPa})$ & $C$ \\
\hline 983 & 0 & 348 & 0 & 0.024 \\
$n$ & $q$ & $\dot{\varepsilon}_{0}\left(\mathrm{~s}^{-1}\right)$ & $T_{0}(\mathrm{~K})$ & $T_{m}(\mathrm{~K})$ \\
\hline 0.32 & 0.69 & 0.1 & 293 & 1943 \\
\hline
\end{tabular}

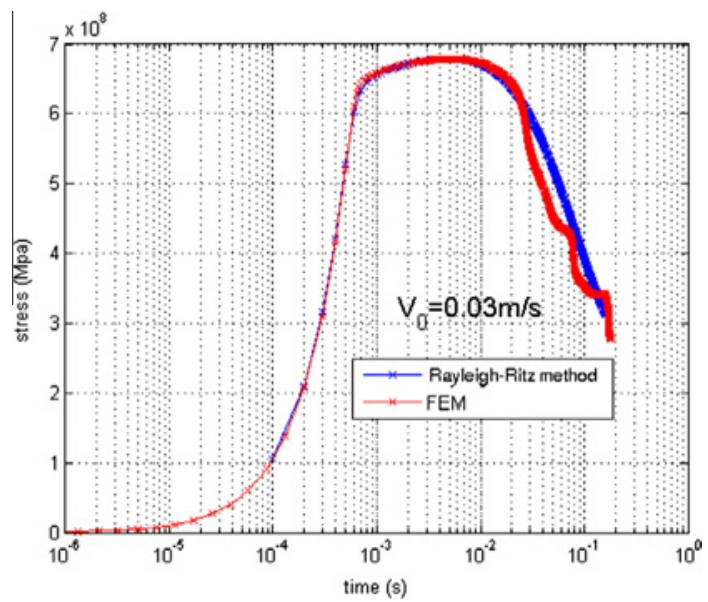

Fig. 14. Evolution of shear stress for $\mathrm{JC}$ model $\left(V_{0}=0.03 \mathrm{~m} / \mathrm{s}\right)$. 


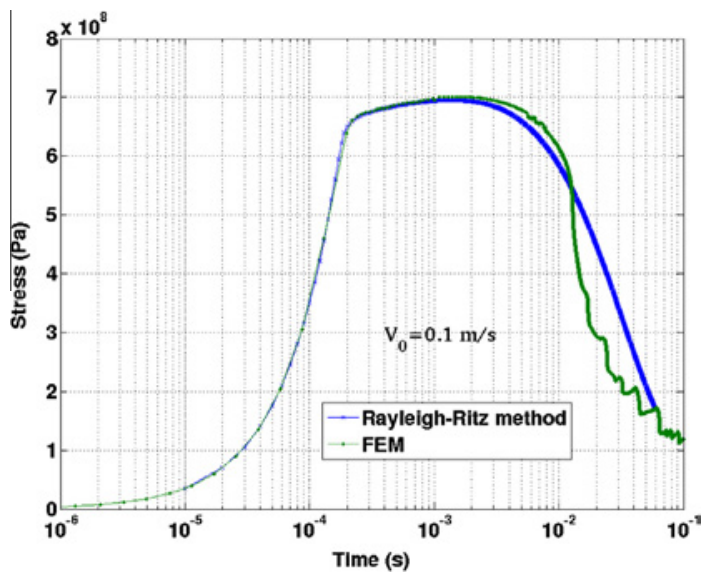

Fig. 15. Evolution of shear stress for $\mathrm{JC}$ model $\left(V_{0}=0.1 \mathrm{~m} / \mathrm{s}\right)$.

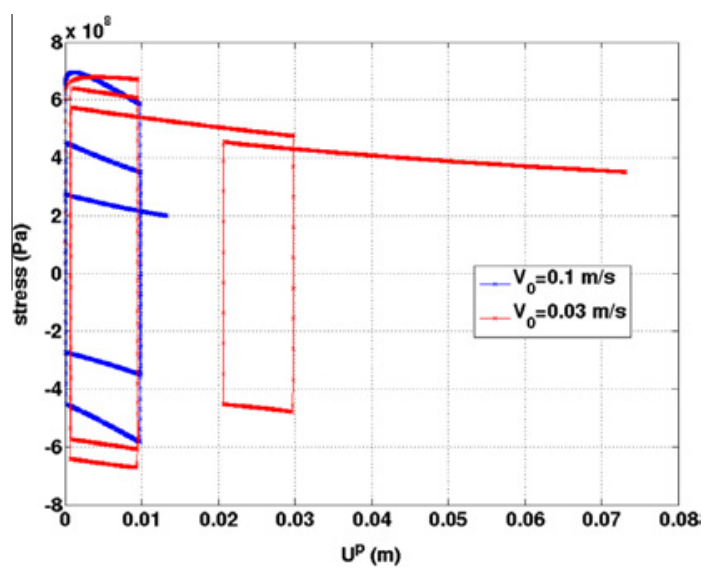

Fig. 16. Shear stress evolution under cyclic loading for JC model $\left(V_{0}=0.03 \mathrm{~m} / \mathrm{s}\right.$ and $\left.V_{0}=0.1 \mathrm{~m} / \mathrm{s}\right)$.

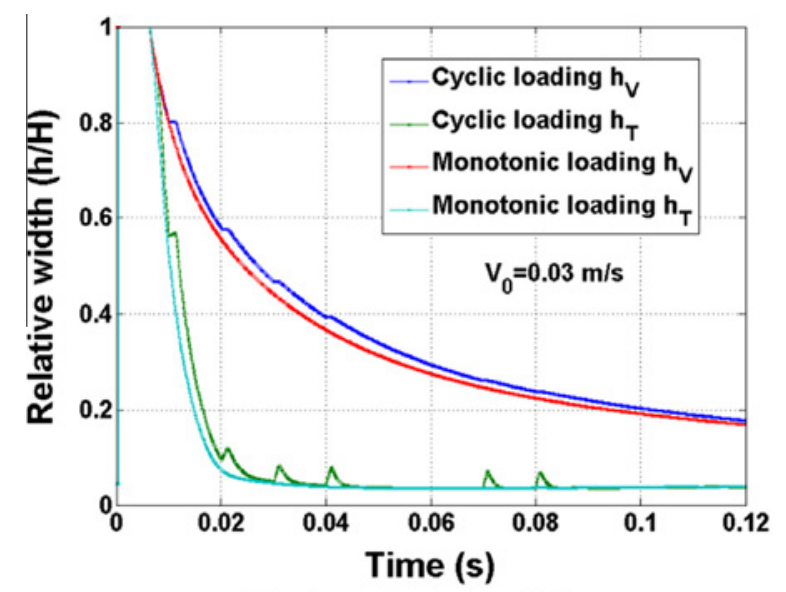

(a) shear band widths tion to a JC thermo-visco-plasticity model, with a set of material parameters corresponding to a $\mathrm{Ti}-6 \mathrm{Al}-4 \mathrm{~V}$ alloy, listed in Table 3 . Note that this choice of parameters ( $A=A_{d}, B=B_{d}$ ) corresponds to a model where the whole plastic work is transformed int heat (Taylor-Quinney coefficient equal to $100 \%$ ). Other choices are possible, with potential effects on shear band formation and evolution, but this aspect will not be investigated here.

Fig. 14 shows the evolution of shear stress, as obtained both by the Ritz-Galerkin formulation and by a FE approach, for a shearing velocity $V_{0}=0.03 \mathrm{~m} / \mathrm{s}$. Once more, we have used a semi-logarithmic plot, in order to emphasize the elastic loading part. Similarly, Fig. 15 shows the evolution of shear stress for a shearing velocity $V_{0}=0.1 \mathrm{~m} / \mathrm{s}$. We can observe a good agreement between the Ritz-Galerkin formulation and the FE approach. Note that the latter is subject to oscillations in the final stages, which can be associated to convergence difficulties (and maybe an unsufficiently refined mesh in the localized shear zone). Note also that, due to the presence of hardening, no steady-state regime can be observed in this case.

In order to test the loading/unloading criterion obtained for the whole shear band in the variational approach, we consider the following cyclic loading:

$U_{0, n+1}=U_{0, n}+\Delta t V_{0}$ for $0 \leqslant t \leqslant 0.1 \mathrm{~s}$,

except for

$U_{0, n+1}=U_{0, n}-\Delta t V_{0}$ for $\left\{\begin{array}{l}0.01 \mathrm{~s}<t \leqslant 0.02 \mathrm{~s} \\ 0.03 \mathrm{~s}<t \leqslant 0.04 \mathrm{~s} \\ 0.07 \mathrm{~s}<t \leqslant 0.08 \mathrm{~s}\end{array}\right.$

The resulting shear stress evolution, for shearing velocities $V_{0}=0.03 \mathrm{~m} / \mathrm{s}$ and $V_{0}=0.1 \mathrm{~m} / \mathrm{s}$, are illustrated at Fig. 16 . In this figure, the stress is plotted versus the plastic part of the displacement jump $U_{0}^{p}$. Note that, as expected, softening occurs earlier under higher shear velocity. Fig. 17 shows the associated evolution of shear band width and

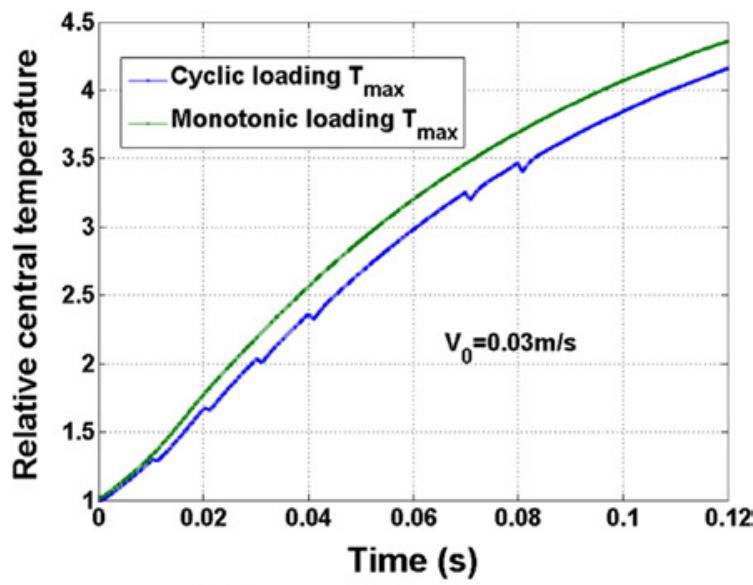

(b) central temperature

Fig. 17. Evolution of shear band widths under cyclic and monotonous loading for $\mathrm{JC}$ model $\left(V_{0}=0.03 \mathrm{~m} / \mathrm{s}\right)$. 


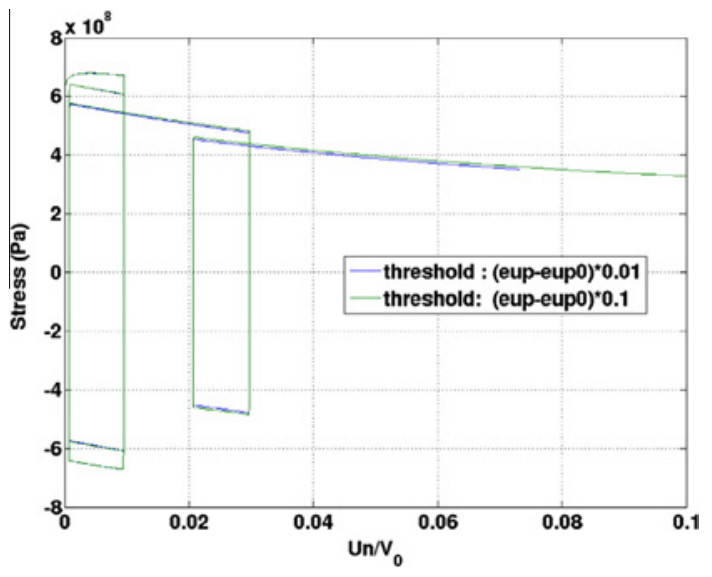

Fig. 18. Influence of $\epsilon$ on stress evolution under cyclic loading for JC model $\left(V_{0}=0.03 \mathrm{~m} / \mathrm{s}\right)$.

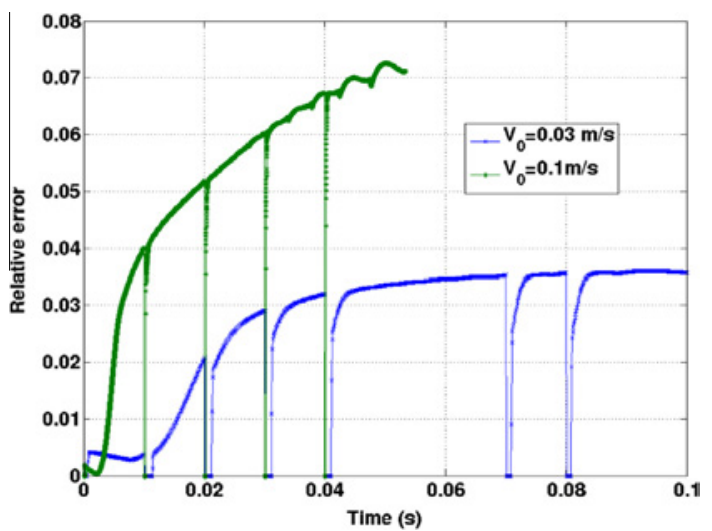

Fig. 19. Approximation error on average yield stress under cyclic loading for JC model. central temperature parameters, with a comparison of cyclic and equivalent monotonous loading. We can observe short plateaux in the evolution of $h_{V}$, corresponding to elastic unloading phases. Regarding temperature, heat diffusion dominates during these elastic unloading phases, which corresponds to an increase of $h_{T}$, accompanied by a decrease of $T_{\max }$. This diffusion effect becomes more marked as the shear band intensifies, since temperature gradients become stronger.

In the algorithm discussed at the end of Section 3.1, we introduced a tolerance $\epsilon$ on the increment of $U_{0}^{p}$ which controls the convergence of the iterative loop. Fig. 18 shows stress-displacement jump curves obtained with two values of this parameter: $\epsilon=0.01$ and $\epsilon=0.1$. We can observe very little difference between the two curves, indicating that a value of $\epsilon=0.1$ is typically sufficient to ensure a decent precision in our case. This loose tolerance may seem surprising, but it is linked to the fact that we are lead to use small time increments to ensure more global convergence. A tighter tolerance $\epsilon$ might be needed if we could use larger time steps (which is not the case for this specific model and loading rate).

Finally, we can try to estimate the error resulting from the use of the average yield criterion $|\tau| \leqslant \bar{\tau}_{y}$ stemming from the variational formulation (see Appendix A):

$\bar{\tau}_{y}=\frac{1}{2 \sqrt{3}} \int_{-H}^{H} \frac{1-\tanh \left(y / h_{V_{n+1}}\right)}{h_{V_{n+1}} \tanh \left(H / h_{V_{n+1}}\right)}\left(\frac{\partial W^{p}}{\partial \bar{\varepsilon}^{p}}+\frac{T_{n+1}}{T_{n}} \frac{\partial \psi^{*}}{\partial \dot{\bar{\varepsilon}}^{p}}\right) d y$.

Given our approximations, the elastic shear stress is constant across the band thickness:

$\tau=\mu \frac{U_{0}^{e}}{H}$

and our global yield criterion is based on the comparison of this stress with the average yield shear stress $\bar{\tau}_{y}$. Locally (at each $y$ ), the actual yield shear stress is given by

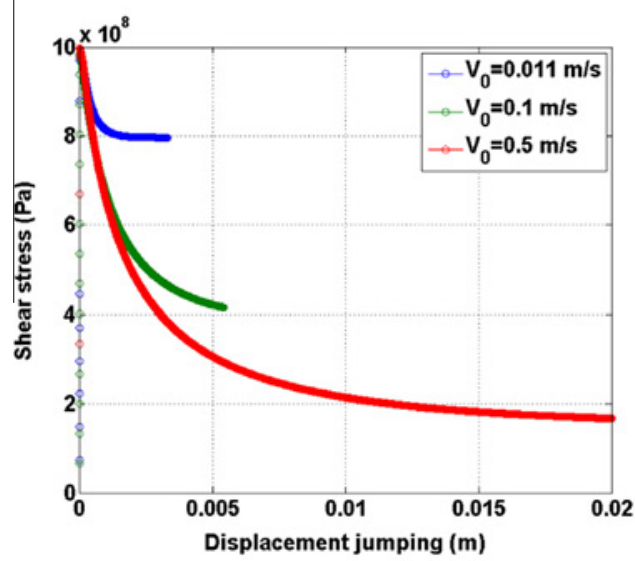

(a) traction-separation

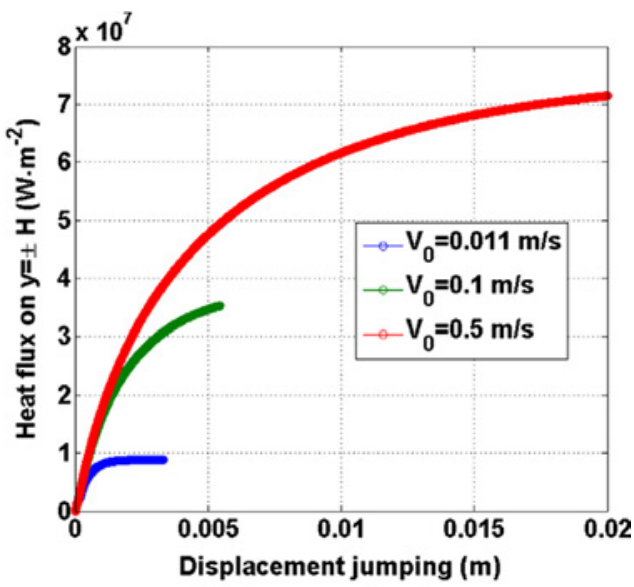

(b) heat production

Fig. 20. Traction-separation (a) and heat production (b) curves for exponential thermal softening model. 
$\tau_{y}(y)=\frac{1}{\sqrt{3}}\left(\frac{\partial W^{p}}{\partial \overline{\bar{\varepsilon}}^{p}}+\frac{T_{n+1}}{T_{n}} \frac{\partial \psi^{*}}{\partial \dot{\bar{\varepsilon}}^{p}}\right) d y$.

We can then estimate the relative error on the yield criterion at a given time by

$e^{*}=\max _{y}\left|1-\frac{\tau_{y}(y)}{\bar{\tau}_{y}}\right|$.

Fig. 19 plots the evolution of this error, for cyclic loading with $V_{0}=0.03 \mathrm{~m} / \mathrm{s}$ and $V_{0}=0.1 \mathrm{~m} / \mathrm{s}$. We can observe that this error accumulates as the loading increases. Under small shearing velocity $\left(V_{0}=0.03 \mathrm{~m} / \mathrm{s}\right)$, the error remains bounded under $4 \%$, while it is larger under faster shearing velocity $\left(V_{0}=0.1 \mathrm{~m} / \mathrm{s}\right)$. Note that the weighting function appearing in (65) gives more importance to values of the effective yield stress in the localized shear zone. In practice, this error will also be affected by the precision of the numerical integration required to evaluate (65) (a compromise must be adopted between precision and efficiency).

\section{Conclusion}

Starting from the variational formulation of coupled thermo-mechanical boundary-value problems of Yang et al. (2006), and using canonical functions established by Leroy and Molinari (1992), we have proposed a novel variational Ritz-Galerkin approach for modeling adiabatic shear band structure evolution, including elasticity, work hardening, and heat conduction. We have first considered the case of non-hardening thermo-visco-plasticity, and then extended the approach to general hardening thermo-visco-plasticity, illustrating our approach on the popular Johnson-Cook model. This approach allows to model the formation of adiabatic shear bands (ASB), with a transition from a state of uniform plastic strain rate and temperature to sharply localized profiles of these quantities. The inclusion of elasticity allows for nonmonotonous loading, the variational formulation providing a global plastic loading/elastic unloading criterion involving an average yield stress over the shear band width. The proposed Ritz-Galerkin approach has been validated by comparison with finite element simulations of the simple shear problem. Note that while such a finite element model typically involves hundreds of degrees of freedom, the Ritz-Galerkin approach only involves four scalar unknowns, leading to a better numerical efficiency for comparable results.

Adiabatic shear bands occurring in 2D or 3D forming or impact problems have been modeled through interface cohesive elements (e.g. Yang et al., 2005) or XFEM enrichement (e.g. Areias and Belytschko, 2007). These approaches are based on assumptions of a uniform plastic strain-rate and a temperature profile directly parameterized by the layer (half-)width $H$. In these models, the adiabatic shear band behavior is thus strongly dependent on the choice of $H$. In our model, although the dependency on $H$ is not completely removed, the width(s) $h_{V, T}$ of the localization zone is (are) computed at each time step. Note that some solutions aiming at removing this dependence have been explored in Su et al. (2014) (see also Su, 2012). Thus, the proposed model could be used to improve interface element, XFEM enrichment, or embedded discontinuities approaches by introducing a better model of the ASB. In other words, it can be seen as a way to provide a physically-motivated thermo-mechanical shear discontinuity model, with ratedependent traction and heat production laws in function of the displacement jump, as illustrated in Fig. 20. Finally, note that the intrinsically 1D nature of the fine scale model used to derive this thermo-mechanical shear discontinuity model prevents to describe precisely the very early stages of ASB initiation and propagation (i.e. the ASB tip). Modeling this transition zone would require to include a 2D (at least) kinematics like in Gioia and Ortiz (1996) or Mercier and Molinari (1998).

\section{Appendix A. Derivation of average yield condition}

In the absence of hardening (Section 3), and given the assumptions adopted for the unidimensional shear band model, the functional to be minimized in variational principle (22) reduces to

$$
\begin{aligned}
J_{n}\left(V_{0}, V_{0}^{p}, h_{V_{n+1}}, T_{\max _{n+1}}, h_{T_{n+1}}\right)= & {\left[W^{e}\left(U_{0_{n+1}}^{e}\right)-W^{e}\left(U_{0_{n}}^{e}\right)\right.} \\
& \left.+\Delta t \psi^{*}\left(\frac{T_{n+1}(y)}{T_{n}(y)} \frac{1}{\sqrt{3}}\left|\dot{\gamma}^{p}(y)\right|\right)\right] \\
& +W^{t}\left(T_{n+1}(y)\right)-W^{t}\left(T_{n}(y)\right)
\end{aligned}
$$

where we have adopted (for simplicity) the choice $\alpha=0$ in (24), with $\dot{\gamma}^{p}(y)$ and $T(y)$ respectively given by (45) and (44), and with

$U_{0_{n+1}}^{e}=U_{0_{n}}^{e}+\left(V_{0}-V_{0}^{p}\right) \Delta t$.

The functional to optimize in the shear band Ritz-Galerkin variational principle is then given by

$$
\begin{aligned}
\Phi_{n}\left(V_{0}, V_{0}^{p}, h_{V_{n+1}}, T_{\max _{n+1}}, h_{T_{n+1}}\right) & =\int_{-H}^{H}\left[J_{n}\left(V, V_{0}^{p}, h_{V_{n+1}}, T_{\max _{n+1}}, h_{T_{n+1}}\right)\right. \\
& \left.-\Delta t \mathcal{X}\left(\boldsymbol{G}\left(T_{\max _{n+1}}, h_{T_{n+1}}\right)\right)\right] d y, \quad(A,),
\end{aligned}
$$

where $\boldsymbol{G}\left(T_{\max _{n+1}}, h_{T_{n+1}}\right)$ is obtained by inserting (44) in the definition (28). The derivative of this functional with respect to $V_{0}^{p}$ is then given by

$$
\begin{aligned}
\frac{\partial \Phi_{n}}{\partial V_{0}^{p}=} & -2 H \Delta t \frac{\partial W^{e}}{\partial U_{0}^{e}}+\frac{\Delta t}{\sqrt{3}} \int_{-H}^{H} \operatorname{sign}\left(\dot{\gamma}^{p}\right) \frac{T_{n+1}(y)}{T_{n}(y)} \frac{\dot{\gamma}^{p}(y)}{V_{0}^{p}} \frac{\partial \psi^{*}}{\partial \overline{\bar{\varepsilon}}^{p}} \\
& \left(\frac{T_{n+1}(y)}{T_{n}(y)} \frac{1}{\sqrt{3}}\left|\dot{\gamma}^{p}(y)\right|\right) d y \\
= & -2 \Delta t \mu \frac{U_{0_{n+1}^{e}}^{e}}{H}+\frac{\Delta t}{\sqrt{3}} \int_{-H}^{H} \operatorname{sign}\left(\dot{\gamma}^{p}\right) \frac{T_{n+1}(y)}{T_{n}(y)} \frac{\dot{\gamma}^{p}(y)}{V_{0}^{p}} \frac{\partial \psi^{*}}{\partial \dot{\bar{\varepsilon}}^{p}} \\
& \left(\frac{T_{n+1}(y)}{T_{n}(y)} \frac{1}{\sqrt{3}}\left|\dot{\gamma}^{p}(y)\right|\right) d y \\
= & -2 \Delta t\left[\tau-\operatorname{sign}\left(V_{0}^{p}\right) \bar{\tau}_{y}\right],
\end{aligned}
$$

where

$\tau=\mu \frac{U_{0_{n}}^{e}+\left(V_{0}-V_{0}^{p}\right) \Delta t}{H}$ 
and

$$
\begin{aligned}
\bar{\tau}_{y}= & \frac{1}{2 \sqrt{3}} \int_{-H}^{H} \frac{T_{n+1}(y)}{T_{n}(y)} \frac{1-\tanh \left(y / h_{V_{n+1}}\right)}{h_{V_{n+1}} \tanh \left(H / h_{V_{n+1}}\right)} \frac{\partial \psi^{*}}{\partial \dot{\bar{\varepsilon}}^{p}} \\
& \left(\frac{T_{n+1}(y)}{T_{n}(y)} \frac{1}{\sqrt{3}}\left|\dot{\gamma}^{p}(y)\right|\right) d y .
\end{aligned}
$$

When computing the elastic predictor, one has $V_{0}^{p}=0, \dot{\gamma}^{p}(y)=0$ and $T_{n+1}(y)=T_{n}(y)$, thus leading to

$\tau_{\mathrm{tr}}=\mu \frac{U_{0_{n}}^{e}+V_{0} \Delta t}{H}$ and

$\bar{\tau}_{y}=\frac{1}{2 \sqrt{3}} \int_{-H}^{H} \frac{1-\tanh \left(y / h_{V_{n+1}}\right)}{h_{V_{n+1}} \tanh \left(H / h_{V_{n+1}}\right)} \frac{\partial \psi^{*}}{\partial \dot{\bar{\varepsilon}}^{p}}(0) d y$

the latter expression yielding (49) when $\psi^{*}$ is given by (39).

In the presence of hardening (Section 4), the same reasoning applies, with

$$
\begin{gathered}
\frac{\partial J_{n}}{\partial \Delta U_{0}^{p}}=-\frac{\partial W^{e}}{\partial U_{0}^{e}}+\operatorname{sign}\left(\Delta U_{0}^{p}\right) \frac{1}{\sqrt{3}} \frac{1-\tanh \left(y / h_{V_{n+1}}\right)}{h_{V_{n+1}} \tanh \left(H / h_{V_{n+1}}\right)} \\
\left(\frac{\partial W^{p}}{\partial \overline{\bar{\varepsilon}}^{p}}+\frac{T_{n+1}}{T_{n}} \frac{\partial \psi^{*}}{\partial \overline{\bar{\varepsilon}}^{p}}\right)
\end{gathered}
$$

and we can thus define the average yield stress as

$$
\bar{\tau}_{y}=\frac{1}{2 \sqrt{3}} \int_{-H}^{H} \frac{1-\tanh \left(y / h_{V_{n+1}}\right)}{h_{V_{n+1}} \tanh \left(H / h_{V_{n+1}}\right)}\left(\frac{\partial W^{p}}{\partial \overline{\bar{\varepsilon}}^{p}}+\frac{T_{n+1}}{T_{n}} \frac{\partial \psi^{*}}{\partial \dot{\bar{\varepsilon}}^{p}}\right) d y .
$$

\section{Appendix B. Finite element formulation of the unidimensional shear band problem}

A detailed description of the incremental variational formulation of general thermo-visco-plasticity can be found in Stainier (2013), for example. If we particularize to the case of the model shear band considered here, it reduces to (31):

$$
\inf _{u_{n+1} \sup _{n+1}} \int_{-H}^{H}\left[\mathcal{W}_{n}\left(\gamma_{n+1}, T_{n+1}\right)-\Delta t \chi\left(-\frac{T_{n+1, y}}{T_{n+1}}\right)\right] d y,
$$

where

$$
\begin{aligned}
\mathcal{W}_{n}\left(\gamma_{n+1}, T_{n+1}\right)= & \inf _{\gamma_{n+1}^{p}}\left[W\left(\gamma_{n+1}, T_{n+1}, \gamma_{n+1}^{p}\right)-W\left(\gamma_{n}, T_{n}, \gamma_{n}^{p}\right)\right. \\
& -\rho_{0} \eta_{n}\left(T_{n+1}-T_{n}\right) \\
& \left.+\int_{t_{n}}^{t_{n+1}} \psi^{*}\left(\frac{T_{n+1}}{T_{n}} \frac{\Delta \gamma^{p}}{\Delta t} ; T(t)\right) d t\right]
\end{aligned}
$$

with the shear strain $\gamma(y)=u_{y}(y)$. A time-discrete incremental finite element formulation can then be derived in a straightforward procedure.

Considering the symmetry of the sought solution, we will define the numerical model on the unidimensional domain $y \in[0, H]$, with boundary conditions

$$
\begin{aligned}
u(0, t) & =0, T_{y}(0, t)=0 \text { and } u(H, t) \\
& =U_{0}\left(t_{n+1}\right), T(H, t)=T_{0},
\end{aligned}
$$

where $U_{0}(t)$ and $T_{0}$ are imposed displacement and temperature at the shear band boundary. We then introduce finite element type approximations of the displacement and temperature fields:

$$
\begin{aligned}
& u(y, t)=\sum_{a=1}^{N} U^{(a)}(t) N_{a}(y), \\
& T(y, t)=\sum_{a=1}^{N} T^{(a)}(t) N_{a}(y),
\end{aligned}
$$

where $U^{(a)}$ and $T^{(a)}$ are nodal displacements and temperatures, and $N_{a}(y)$ interpolating shape functions (i.e. with delta-Dirac properties). In practice, we have considered piecewise linear interpolation. Note also that we have chosen to use the same spatial discretization and shape functions for both displacement and temperature fields.

The variational principle (B.1) then becomes a nonlinear algebraic optimization problem:

$\inf _{\left\{U_{n+1}\right\}} \sup _{\left\{T_{n+1}\right\}} \Phi_{n}\left(\left\{U_{n+1}\right\},\left\{T_{n+1}\right\}\right)$

where $\left\{U_{n+1}\right\}=\left\{U^{(1)}\left(t_{n+1}\right), \ldots, U^{(N)}\left(t_{n+1}\right)\right\}$ and $\left\{T_{n+1}\right\}=$ $\left\{T^{(1)}\left(t_{n+1}\right), \ldots, T^{(N)}\left(t_{n+1}\right)\right\}$ are arrays of nodal displacements and temperatures. This optimization problem, which has to be solved at each time step, can be solved by a standard Newton-Raphson procedure, complemented with a linesearch to improve convergence.

\section{References}

Areias, P.M.A., Belytschko, T., 2007. Two-scale method for shear bands: thermal effects and variable bandwidth. Int. J. Numer. Methods Eng. 72, 658-696.

Biot, M., 1958. Linear thermodynamics and the mechanics of solids. In: Proceedings of the Third US National Congress of Applied Mechanics. ASME, pp. 1-18.

Clifton, R., Molinari, A., 1983. Localisation de la déformation viscoplastique en cisaillement simple: résultats exacts en théorie non linéaire. C.R. Acad. Sci. Paris 2, 1-4.

Dolinski, M., Rittel, D., Dorogoy, A., 2010. Modeling adiabatic shear failure from energy considerations. J. Mech. Phys. Solids 58 (11), 1759-1775.

Gioia, G., Ortiz, M., 1996. The two-dimensional structure of dynamic boundary layers and shear bands in thermoviscoplastic solids. J Mech. Phys. Solids 44 (2), 251-292.

Halphen, B., Nguyen, Q.S., 1975. Sur les matériaux standard généralisés. J. Méc. 14 (1), 39-63.

Johnson, G.R., Cook, W.H., 1983. A constitutive model and data for metals subjected to large strains, high strain rates and high temperatures. In: Proceedings of the Seventh International Symposium on Ballistics. The Hague, pp. 541-547.

Leroy, Y.M., Molinari, A., 1992. Stability of steady states in shear zones. J. Mech. Phys. Solids 40 (1), 181-212.

Li, S., Liu, W.K., Rosakis, A.J., Belytschko, T., Hao, W., 2002. Mesh-free Galerkin simulations of dynamic shear band propagation and failure mode transition. Int. J. Solids Struct. 39 (5), 1213-1240.

Longère, P., Dragon, A., Trumel, H., de Resseguier, T., Deprince, X., Petitpas, E., 2003. Modelling adiabatic shear banding via damage mechanics approach. Arch. Mech. 55, 3-38.

Longère, P., Dragon, A., Trumel, H., Deprince, X., 2005. Adiabatic shear banding induced degradation in a thermo-elastic/viscoplastic material under dynamic loading. Int. J. Impact Eng. 32, 285-320.

Longère, P., Dragon, A., Deprince, X., 2009. Numerical study of impact penetration shearing employing finite strain viscoplasticity model incorporating adiabatic shear banding. ASME J. Eng. Mater. Technol. 131 (1), 011105.

Medyanik, S.N., Liu, W.K., Li, S., 2007. On criteria for dynamic adiabatic shear band propagation. J. Mech. Phys. Solids 55 (7), 1439-1461.

Mercier, S., Molinari, A., 1998. Steady-State shear band propagation under dynamic conditions. J. Mech. Phys. Solids 46 (8), 1463-1495.

Merzer, A., 1982. Modelling of adiabatic shear band development from small imperfections. J. Mech. Phys. Solids 30 (5), 323-338. 
Miehe, C., 1995. Entropic thermoelasticity at finite strains. Aspects of the formulation and numerical implementation. Comput. Methods Appl. Mech. Eng. 120 (3-4), 243-269.

Oliver, J., Huespe, A., 2004. Continuum approach to material failure in strong discontinuity settings. Comput. Methods Appl. Mech. Eng. 193 (30-32), 3195-3220.

Oliver, J., Cervera, M., Manzoli, O., 1999. Strong discontinuities and continuum plasticity models: the strong discontinuity approach. Int. J. Plast. 15, 319-351.

Ortiz, M., Pandolfi, A., 1999. Finite-deformation irreversible cohesive elements for three-dimensional crack-propagation analysis. Int. J. Numer. Methods Eng. 44 (9), 1267-1282.

Ortiz, M., Stainier, L., 1999. The variational formulation of viscoplastic constitutive updates. Comput. Methods Appl. Mech. Eng. 171 (3-4), 419-444.

Ortiz, M., Leroy, Y., Needleman, A., 1987. A finite element method for localized failure analysis. Comput. Methods Appl. Mech. Eng. 61, 189214.

Rittel, D., Landau, P., Venkert, A., 2008. Dynamic recrystallization as a potential cause for adiabatic shear failure. Phys. Rev. Lett. 101 (16), 165501.

Stainier, L., 2011. Consistent incremental approximation of dissipation pseudo-potentials in the variational formulation of thermo- mechanical constitutive updates. Mech. Res. Commun. 38 (4), 315319.

Stainier, L., 2013. A Variational Approach to Modeling Coupled ThermoMechanical Nonlinear Dissipative Behaviors. Advances in Applied Mechanics, vol. 46. Elsevier, pp. 69-126.

$\mathrm{Su}$, S., 2012. Energy-Based Variational Modeling of Adiabatic Shear Band Structure (Ph.D. thesis). Ecole Centrale de Nantes. <http:// tel.archives-ouvertes.fr/tel-00797178>.

Su, S., Stainier, L., Mercier, S., 2014. Energy-based variational modeling of fully formed adiabatic shear bands. Eur. J. Mech. - A/Solids 47, 1-13.

Wright, T.W., 2002. The Physics and Mathematics of Adiabatic Shear Band. Cambridge Monographs on Mechanics. Cambridge University Press.

Wright, T.W., Ravichandran, G., 1997. Canonical aspects of adiabatic shear bands. Int. J. Plast. 13 (4), 309-325.

Yang, Q., Mota, A., Ortiz, M., 2005. A class of variational strain-localization finite elements. Int. J. Numer. Methods Eng. 62 (8), 1013-1037.

Yang, Q., Stainier, L., Ortiz, M., 2006. A variational formulation of the coupled thermo-mechanical boundary-value problem for general dissipative solids. J. Mech. Phys. Solids 54 (2), 401-424.

Zener, C., Hollomon, J.H., 1944. Effect of strain rate upon plastic flow of steel. J. Appl. Phys. 15 (1), 22-32. 